\title{
LES VANNERIES GALLO-ROMAINES Du Petit-Creusot à Chalon-Sur-SaÔne (SAÔNE-ET-LOIRE)
}

\author{
Gérard MONTHEL ${ }^{*}$, Nicole Bl.ANC ${ }^{* *}$ et Françoise GURY ${ }^{* * *}$
}

Mots-clés. Gaule romaine, Saône, Chalon-sur-Saône, méandre, pédologie, artisanat, vannerie, nasse, corbeille, pêche fluviale.

Key-words. Roman Gaul, Saône, Chalon-sur-Saône, meander, pedology, craftsmanship, basketry, fish trap, basket, river fishing.

Résumé. Une fouille de sauvetage dans un méandre fossile de la Saône, au sud de Chalon-sur-Saône, au lieu-dit Le Petit-Creusot, a permis de mettre au jour parmi un abondant mobilier - dont de nombreux textiles - une nasse el une corbeille datables du III ${ }^{\text {s. Les }}$ matériaux employés ont été identifiés : osier, mais aussi clématite et cornouiller pour la corbeille. Le bon état de la nasse, intégralement conservée à l'exception de la jointure du corps et du goulet, a permis d'en déterminer le mode de construction, confirmé par l'archéologie expérimentale. Ces données, jointes à l'apport des textes et de l'iconographie, permellent d'éclairer usage et conditions de production de ces pièces. Ces découvertes apportent une contribution capitale à notre connaissance de la vannerie en Gaule romaine, jusqu'à maintenant pauvre en vestiges, et éclairent d'un jour nouveau le faciès de la rivière dans cette zone.

\begin{abstract}
A rescue excavation on the bank of a fossil meander of the Saône river, south of Chalon-sur-Sâne in the locality of PetitCreusot, revealed a fish trap and a basket among a large amount of artifacts, including rumerous textiles. The materials employed were identified as willow, plus clematis and dogwood for the basket. Thanks to the good state of preservation of the fish trap, with the exception of the junction of the body and neck, it has been possible to determine weaving processes which were confirmed through experimentation. These data, coupled with information from wrilten sources and iconographic evidence, permit a better understanding of the utilization and conditions of production of these objects. These discoveries are a major contribution to our knowledge of basketry in Roman Gaul which until now left little trace, and provide new information concerning the river facies in this area.
\end{abstract}

\section{CIRCONSTANCES DE LA DÉCOUVERTE}

Les vanneries gallo-romaines qui font l'objet de cette étude ont été mises au jour en décembre 1985, à

* UMR 7055 du CNRS, Préhistoire et technologie, Maison RenéGinouvès, 21 allée de l'Université, F-92023 Nanterre cedex. Mél. : monthel@mae.u-paris10.fr

** EP 2046 du CNRS, LIMC, Maison René-Ginouvès, 21 allée de l'Université, F-92023 Nanterre cedex. Mél. : blancn@mac.u-paris10.fr

*** UMR 126 du CNRS, Archéologies d'Orient et d'Occident, École normale supérieure, 45 rue d'Ulm, F-75230 Paris cedex 0.5 . l'emplacement des anciens ateliers Creusot-Loire, au lieu-dit Le Petit-Creusot ${ }^{1}$ (fig. 1).

À environ $100 \mathrm{~m}$ de la berge et $150 \mathrm{~m}$ en amont du pont des Dombes, une suite de strates riches en mobilier archéologique fut découverte, entre $6,50 \mathrm{~m}$ et $8,47 \mathrm{~m}$ de profondeur, lors de travaux dans l'enceinte d'une entreprise dont l'activité particulièrement sensible touche à la

1. Cf. Monthel, 1987, 1987-1988, 1996. L'endroit était déjà connu par la découverte en 1912 d'une nécropole à incinération, mise au jour lors de la construction d'un bâtiment de l'usine Schneider (le bâtiment des chaudières). 


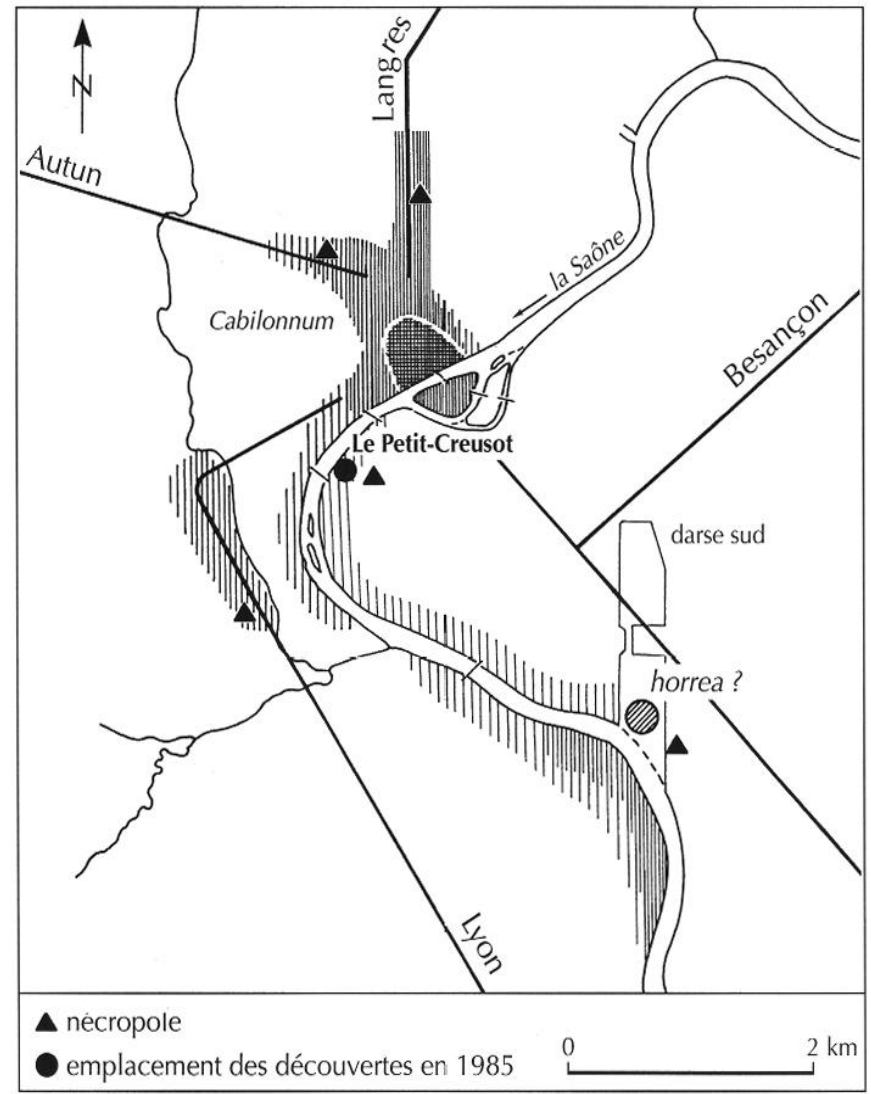

Fig. 1 - Le site du Petit-Creusol, Chalon-sur-Saône : trame croisée, castrum $d u I V^{*}$ s. ; trame verticale serrée, habilal antique assuré; trame verticale lâche, habilal antique probable (dessin C. Monthel).

recherche nucléaire. Avertis tardivement ${ }^{2}$ de la mise au jour de ces vestiges qui s'étageaient sur près de $2 \mathrm{~m}$ d'épaisseur, nous dûmes limiter notre intervention à une surface de $20 \mathrm{~m}^{2}$ environ, qui se trouvait encore partiellement en place. La fouille se déroula pendant l'hiver 1985-1986, dans des conditions climatiques difficiles ${ }^{3}$ et surtout avec pompage incessant des eaux d'infiltration, puisque le niveau de la rivière très proche est situé à - 4,80 m. La stratigraphie, de $-5,40 \mathrm{~m}$ à $-8,47 \mathrm{~m}$, se composait d'une succession de 22 couches d'argiles fines et de sables reposant sur un socle de gravier (fig. 2). Celles-ci attestent l'activité de la rivière, à l'emplacement

2. Par le conservateur de la section archéologique du musée Denon de Chalon-sur-Saône, que nous remercions.

3. I a fouille eut lieu entre le 25 décembre 1985 et le $1^{\mathrm{cr}}$ janvicr 1986. L'eau utilisée pour la fabrication de la coque en plâtre de la nasse gelait spontanément si elle n'était pas chauffée au préalable.

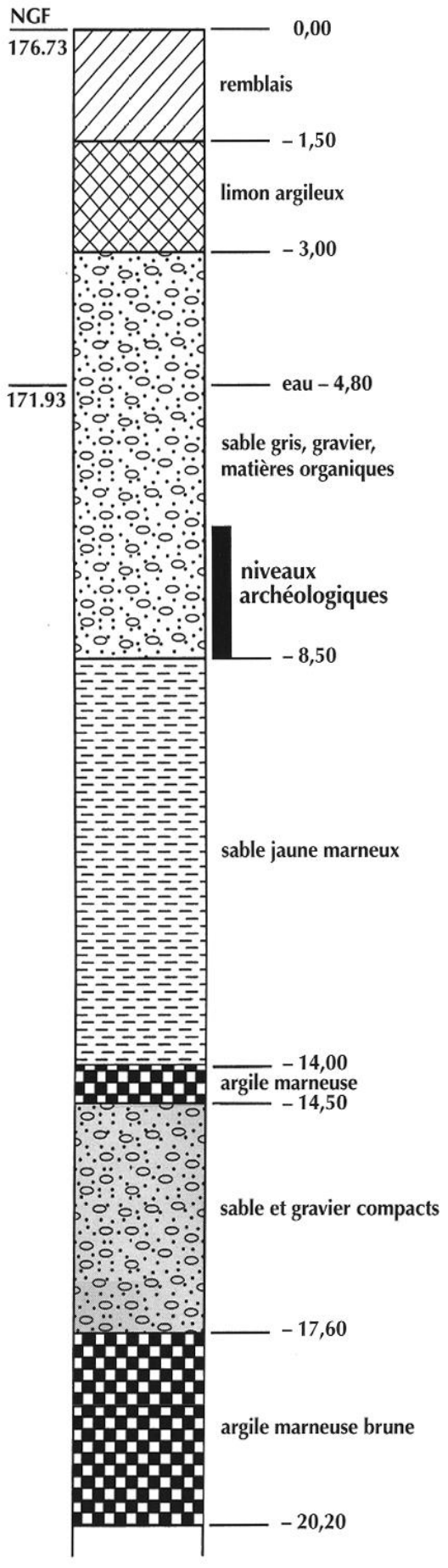

Fig. 2 - Colonne géologique du site du Pelit-Creusot (dessin C. Monthel).

de son lit ${ }^{4}$ : d'abord en eau courante, puis en eau progressivement ralentie entre les niveaux $-8,47 \mathrm{~m}$ et $-7,85 \mathrm{~m}$, et enfin en eau stagnante, ensuite marécageuse,

4. L'étude pédologique préalable de ces différentes couches a été effectuée en 1988 par Jean-Paul Bravard (UMR 183, UMR 5600 du CNRS, université I.yon III). Un litre environ de sédiment a été prélevé 
et pour finir en état asséché avec formation d'une berge, entre les niveaux $-7,85 \mathrm{~m}$ et $-6,50 \mathrm{~m}{ }^{5}$. Entre ces deux variations extrêmes de régime, du mouvement rapide avec dépôt de gravier à l'arrêt d'activité d'un courant (à l'exception de crues épisodiques déposant essentiellement de fines particules argilo-sableuses et limoneuses), s'intercale, aux niveaux $-7,85 \mathrm{~m} /-7,87 \mathrm{~m}$, une mince couche de végétaux partiellement décomposés mêlés de faune malacologique. Sa présence montre l'existence d'une phase d'eau dormante très peu profonde, voire de simple humidité, durant laquelle poussaient joncacées et salicinées. Ce stade est transitoire entre états en eau et hors d'eau, entre rivière et rive.

Malgré la faible surface réellement fouillée (environ la moitié des $20 \mathrm{~m}^{2}$ étudiés), le mobilier mis au jour est important, tant en quantité qu'en qualité.

La céramique était essentiellement répartie dans les couches des états originellement en eaux, les témoins devenant de plus en plus volumineux du haut vers le bas: du simple tesson dans les couches supérieures aux vases intacts dans les couches inférieures (20-22). Quelquesuns fournissent de solides critères de datation : un vase à médaillons d'applique de la vallée du Rhône, dont les motifs bien répertoriés sont ici le résultat de nombreux surmoulages, correspond à une production relativement tardive du III ${ }^{e} s .{ }^{6}$. De même, des fragments de céramique africaine, dont la production est datée de la seconde moitié du II's. et de la première moitié du III's. après J.-C., nous placent dans la même fourchette chronologique ${ }^{7}$.

pour chaque couche. Ces échantillons, stockés dans des récipients munis de fermetures hermétiques, ont été déposés dans les réserves de fouilles de la section archéologique du musée Denon, où ils sont conservés.

5. Entre $6,50 \mathrm{~m}$ et $5,40 \mathrm{~m}$, niveau supérieur de la zone conservée et fouillée, les couches, essentiellement d'argiles vierges de tout mobilier, semblent résulter de différents apports d'alluvions de crues. Au-dessus de la côte $-5,40 \mathrm{~m}$, nous ne savons rien de la stratigraphie, détruite.

6. Type F3 de Desbat, 1982. Le premier médaillon $(\emptyset=87 \mathrm{~mm}$ ) représente Mars debout, en armes, entre deux palmes dressées ; à gauche, un autel; des guirlandes sont attachées au cadre formé d'un bourrelet grossier. Ia qualité de ce médaillon, comme du suivant, est médiocre : surmoulage, retouches grossières $\left(c f . \mathrm{n}^{\circ} 106\right.$ de la classification de Wuilleumier, Audin, 1952). Le second médaillon $(\varnothing=80 \mathrm{~mm})$ représente un combat de gladiateurs : un secutor y affronte un Thrace avec, entre eux, un laniste ; entre la tête de ce dernier et celle du Thrace, à droite, on devine : STA[n]T[es] MISSI (réduction [?] du n ${ }^{\circ} 112$ de la classification de Wuilleumier, Audin, 1952).

7. Sigillée claire A : Hayes $14 \mathrm{~B}$, Hayes $3 \mathrm{C}$, non décorée, Hayes 18 ; sigillée claire $\mathrm{C}$ : un fragment d'Hayes 45 ou 46 ; commune africaine: Hayes 23, Hayes 182 ou 196, Hayes 181 et Hayes 197.
Les amphores, dont une dite gauloise intacte ${ }^{8}$, sont nombreuses ${ }^{9}$. Tous les types de denrées qu'elles contenaient sont identifiables ${ }^{10}$ : vin, huile, garum (originaires de Bétique, Gaule du sud, Tripolitaine, Tingitane). Plusieurs pointes sont encore engorgées de la résine nécessaire à la bonne conservation du vin. Tous ces vestiges témoignent d'une circulation commerciale intensive et privilégiée avec le sud.

Dans toutes les couches, les ossements d'animaux sont nombreux, et prouvent une activité notable d'abattage. Outre les bovins, ovins et porcs, on y trouve également des restes de cheval, cerf, chien et volaille.

Le milieu humide a permis la conservation des matériaux périssables : tissus, cuir, bois. Les tissus se présentaient sous forme de boules visqueuses autour desquelles le sable s'était agglutiné ; leur couleur brune fit croire de prime abord que ces textiles étaient enduits de poix ou bitume et avaient pu être employés au calfatage des bateaux, insérés en force dans les interstices des bordages. Or, cette teinte serait naturelle et il semble qu'il faille désormais revenir sur cette hypothèse; la forme de boule de la plupart des fragments est d'ailleurs incompatible avec cet usage d'éléments de calfatage : ils auraient été aplatis ${ }^{11}$.

Outre de traditionnelles semelles cloutées, plusieurs morceaux de cuir, découpes ou chutes, confirmeraient la présence de bourreliers ou cordonniers à proximité. La peausserie est un artisanat qui s'installe naturellement, pour des raisons techniques, en bordure d'une rivière.

Les instruments en bois sont peu variés ; à l'exception d'un bouchon cylindrique et plat et d'un tampon de

8. À rapprocher du groupe G1 de la classification de F. I aubenheimer (1989), malgré la panse peignée et une lèvre correspondant plus aux exemplaires du groupe G5.

9. Quelques éléments de Dressel 1, une Dressel 20 (marque $C H$ dans un cartouche rectangulaire sur une de ses anses), plusieurs tessons dont une lèvre et une anse d'amphore africaine (Tripolitaine III). Tous les éléments de Dressel 1 étaient sur le fond de gravier ; très roulés, ils ne semblent pas avoir été rejetés à cet endroit, mais beaucoup plus en amont.

10. Certaines contenaient également des graines (millet ?) et un dépôt blanc d'origine inconnue.

11. Ce corpus très important de textiles a fait l'objet d'une restauration et d'une étude par le laboratoire du musée des Tissus de Lyon. La taille du dossier et l'apport qu'il constitue pour la connaissance des textiles gallo-romains obligent à une publication séparée. Voir en préalable à cette étude : Monthel et al., 1998. 
bonde de forme bitronconique, les autres éléments ligneux sont de simples pièces portant parfois les traces des outils qui les ont débitées.

Deux objets exceptionnels exigèrent un travail long mais nécessaire de fouille, puis de consolidation in situ avant leur retrait : une nasse (fig. 3 à 7 ) et une corbeille ${ }^{12}$ (fig. 15 à 17). Ces mesures conservatoires ont permis de les sauver ${ }^{13}$. La nasse est à ce jour le seul exemplaire intégralement conservé. Elle figure désormais dans les collections archéologiques du musée Denon de Chalonsur-Saône.

Les deux vanneries étaient incluses dans les couches inférieures de la stratigraphie, elles furent donc abandonnées lorsque le lieu était en pleine eau (niveau $-8,10 \mathrm{~m}$ ). Leur datation est bien assurée par les formes céramiques du III ${ }^{e}$ s., trouvées dans ces couches. La nasse avait sans aucun doute été déposée par un pêcheur, et non jetée (fig. 3 et 4 ). Son axe longitudinal était, comme il est d'usage, parallèle à celui de la rivière, son goulet d'entrée normalement tourné vers l'aval car le poisson remonte à contre-courant. L'extrémité par laquelle on la vidait ne comportait aucun système de fermeture mais celui-ci pouvait être constitué d'un simple «bouchon " d'herbe serrée qui a pu disparaître avant l'enlisement de la nasse. Flle fut envasée et ensablée assez rapidement puisque son volume a été conservé et n'a été que fort peu écrasé par les limons qui l'ont recouverte.

La corbeille, contrairement à la nasse, fut jetée. Elle gisait sur le sable, le fond tourné vers la surface (fig. 15) ; ses parois avaient disparu, avec l'ourlet de son ouverture. La fouille n'a rien révélé d'un éventuel contenu et il semble qu'elle était vide au moment de son abandon.

12. Sur la fouille, avant leur enlèvement, ces deux objets ont été enrobés avec des bandes de non-tissé humidifiées jusqu'au refus, consolidés avec de la mousse polyvinylique et enchâssés dans une coque en plâtre armé. Ils ont été conservés en chambre froide à une température de $0^{\circ}$ à $+5^{\circ}$ et à un taux d'hygrométrie compris entre 90 et $98 \%$. Les laboratoires contactés refusèrent de traiter ces objets, la longueur de la nasse semblant être alors un obstacle majeur à toute restauration. Seul le laboratoire du Schleswig-Holsteinisches Landesmuseum, dans l'extrême nord de l'Allemagne, accepta de procéder au traitement et à la conservation de l'ensemble des objets en matériaux putrescibles (vanneries et textiles).

13. Avec cet inconvénient qu'il n'a pas été possible de conduire une étude détaillée avant restauration, lacune que pallie en partie la couverture photographique.

\section{IA NASSE}

Il s'agit d'une nasse classique constituée d'une entrée ou goulet, en forme d'entonnoir, et du corps lui-même, grossièrement tronconique, dont l'évasement s'accentue, sans rupture de pente, à $35 \mathrm{~cm}$ environ du fond (fig. 3 à 5 ).

\section{DIMENSIONS}

Longueur conservée : 130/134 cm, soit presque la totalité ; l'entrée a souffert, mais la partie arrachée ne doit pas excéder quelques centimètres.

Diamètre de l'entrée : $53 \mathrm{~cm}$ (horizontalement) sur $17,5 \mathrm{~cm}$ (verticalement); cette grande disproportion entre hauteur et largeur s'explique par le tassement de l'objet. Les nasses étant lestées, un léger aplatissement n'a sans doute pas été ménagé pour assurer sa stabilité au fond de l'eau.

Diamètre du fond : $12 / 13 \mathrm{~cm}$.

Longueur du goulet : $44 \mathrm{~cm}$.

Ces dimensions placent la nasse du Petit-Creusot parmi les exemplaires les plus imposants trouvés à ce jour. À titre de comparaison, la nasse trouvée à Melz-surSeine (Seine-et-Marne), et datée de La Tène II finale, mesurait $1,02 \mathrm{~m}$ de longueur; l'entrée avait gardé sa forme circulaire ; son diamètre était de $27 \mathrm{~cm}$ et celui du fond de $8 \mathrm{~cm}$; un étranglement sous le cône lui donnait une forme d'amphore. Elle n'a malheureusement pas survécu au dégagement (Thomasson, 1992, p. 81, fig. 6, $7,9,10$.

\section{MATÉRIAUX}

Le matériau employé a été identifié par macroscopie et microscopie de quatre échantillons pris sur les montants et la trame de remplissage ${ }^{14}$ : l'un comme l'autre sont en saule (Salix sp.). La variété a pu être précisée grâce aux attaches opposées, et non alternes, caractéristiques de Salis purpurea $\mathrm{L}$., appelé aussi osier rouge ou osier des tonneliers.

14. Nous reprenons ici le rapport rédigé par l'auteur de l'analyse, Anne Dietrich (Art Conservation Service), qui a également déterminé les matériaux de la corbcillc. Cette étude a pu être effectuée grâce à un crédit du Centre régional de recherche archéologique de Bourgogne (CRRAB), que nous remercions. 


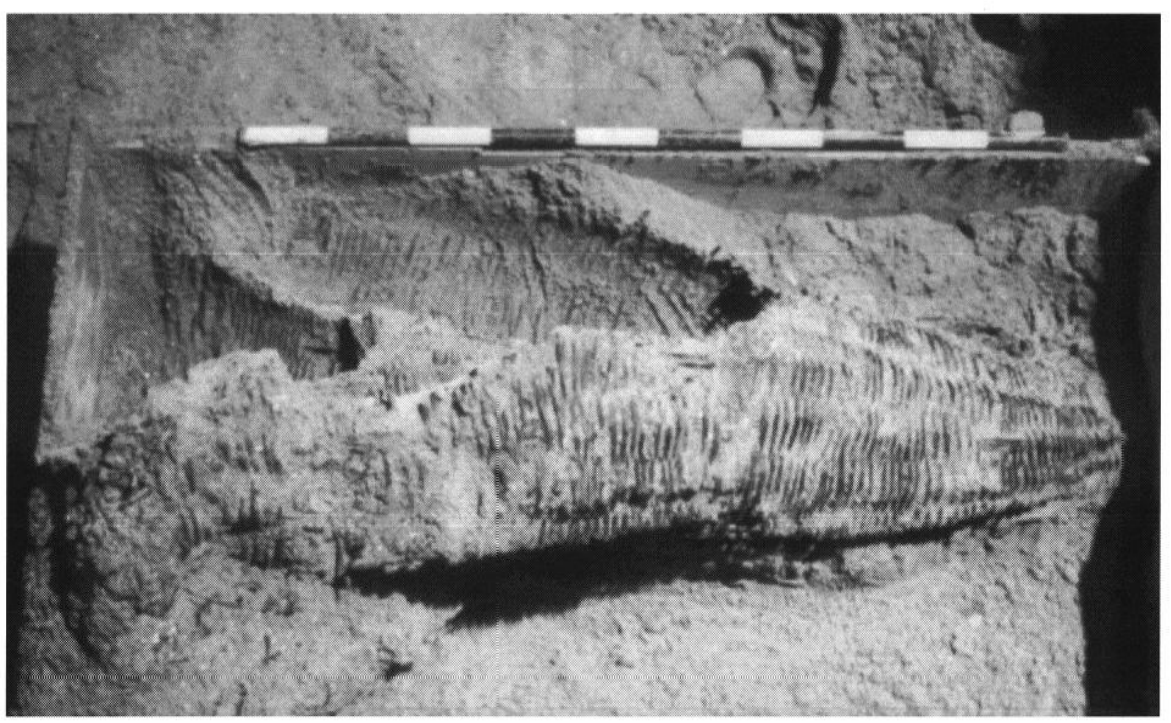

Fig. 3 - La nasse du Petit-Creusot (photo de fouille, cliché G. Monthel).

Selon l'auteur de l'analyse, ce saule n'est pas celui planté par les vanniers (Salix viminalis), mais il est commun au bord de l'eau, des chemins et des bois. C'est une essence de reconquête, utilisée comme tous les saules en vannerie. Beaucoup de brins sont noueux et fourchus. Le matériau n'est pas écorcé : l'écorce à demi détachée est parfois visible (fig. 6 et 7). L'éclatement en fibres de certains brins indique un emploi d'osier vert. Cet éclatement et l'arrachement de la pelure peuvent aussi bien résulter de l'alternance des trempages et des séchages propres à l'utilisation de l'objet que des conditions de conservation jusqu'au moment de la restauration.

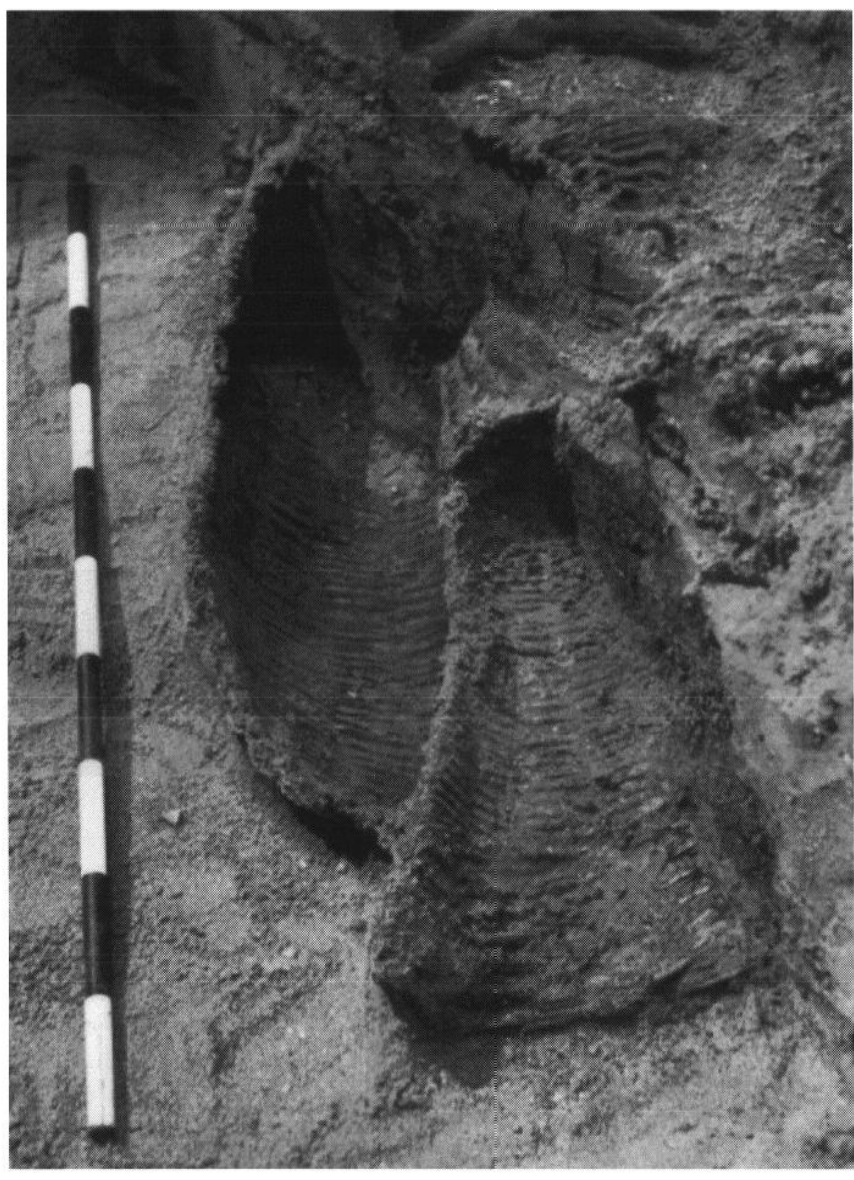

Fig. 4 - La nasse du Petit-Creusot : à droite, goulet d'entrée (photo de fouille, cliché G. Monthel).
Les montants sont constitués de rameaux continus, atteignant jusqu'à $132 \mathrm{~cm}$ de longueur conservée, et employés sans être refendus pour obtenir des éclisses ${ }^{15}$. La partie la plus fine - entre $3 \mathrm{~mm}$ et $6 \mathrm{~mm}$ de section est employée du côté de la sortie étroite, ou fond de la nasse; à l'autre extrémité, les brins peuvent atteindre $8 \mathrm{~mm}$ de section.

\section{CONSTRUCTION}

Les conditions de conservation de l'objet permettent d'observer le corps de la nasse, à l'exception de la partie reposant sur le socle où elle a été installée par le laboratoire de restauration. Elles permettent d'entrevoir, à l'intérieur, le goulet, sans qu'il soit possible de le décrire très précisément.

\section{LE CORPS}

Le corps de la nasse est une réalisation en vannerie de type clayonné, à montants parallèles passifs, autour desquels passent les brins actifs (fig. 5 et 8). Sans pré-

15. En revanche, dans la nasse celtique de Melz-sur-Seine, en saule également (mais sans caractérisation de la variété), on a constaté l'emploi à la fois de brins ronds et de brins refendus (Thomasson, 1992, p. 77). 


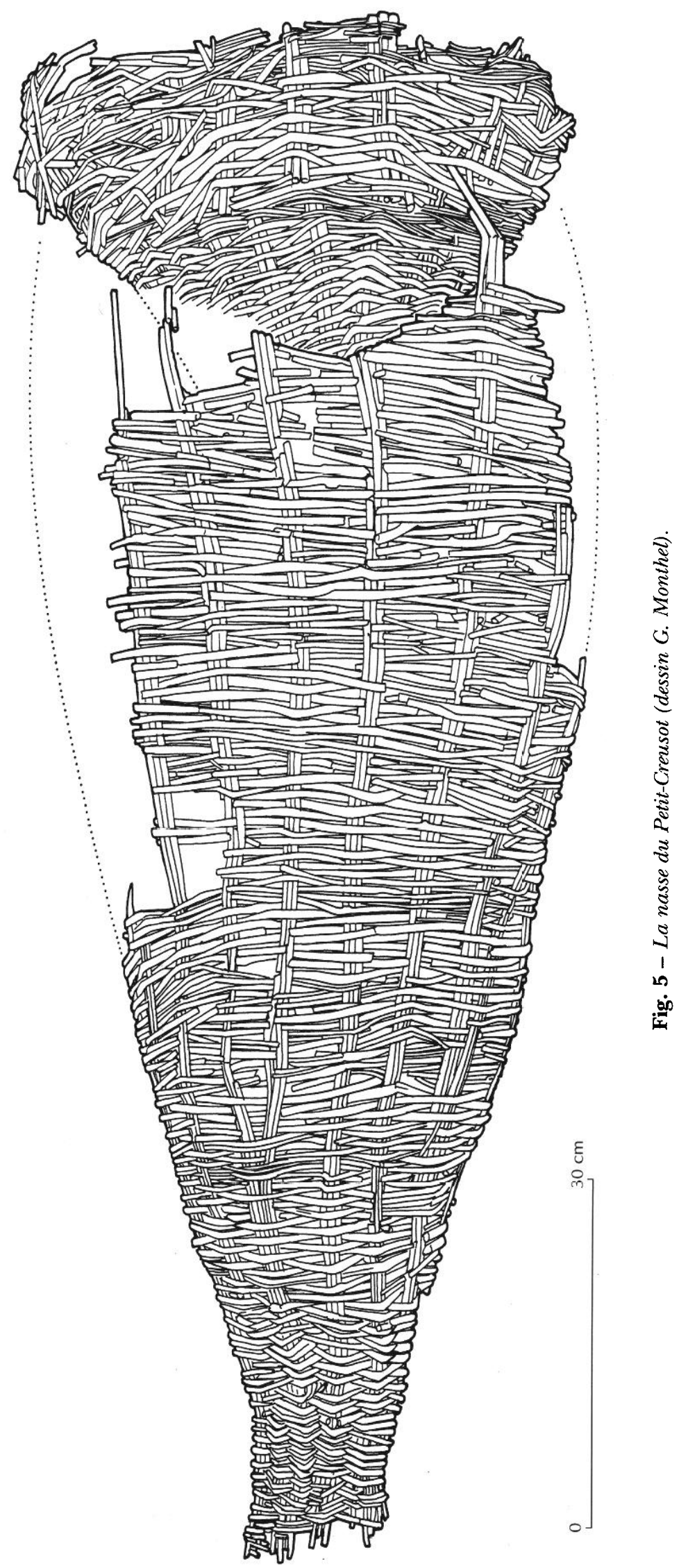




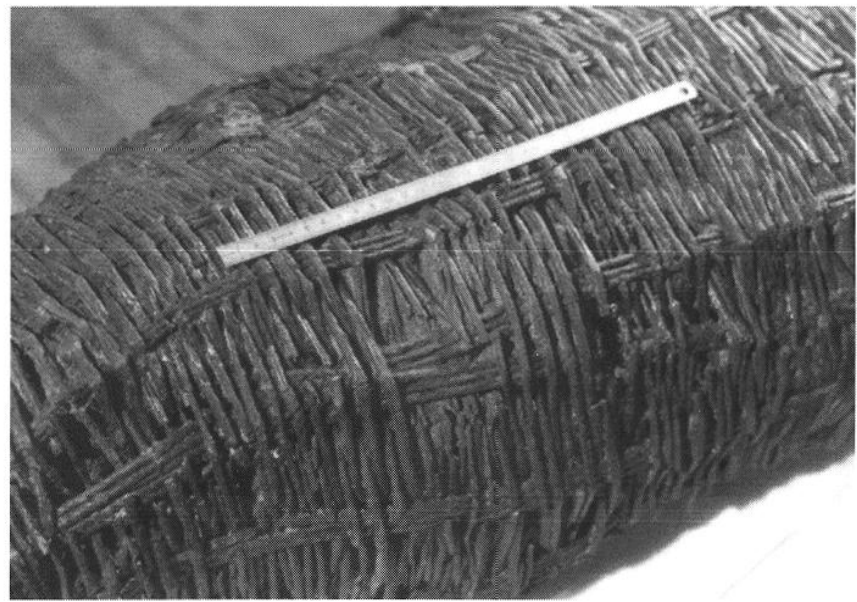

Fig. 6 - Tressage et structure de la nasse du Petit-Creusol (cliché N. Blanc et T: Gury).

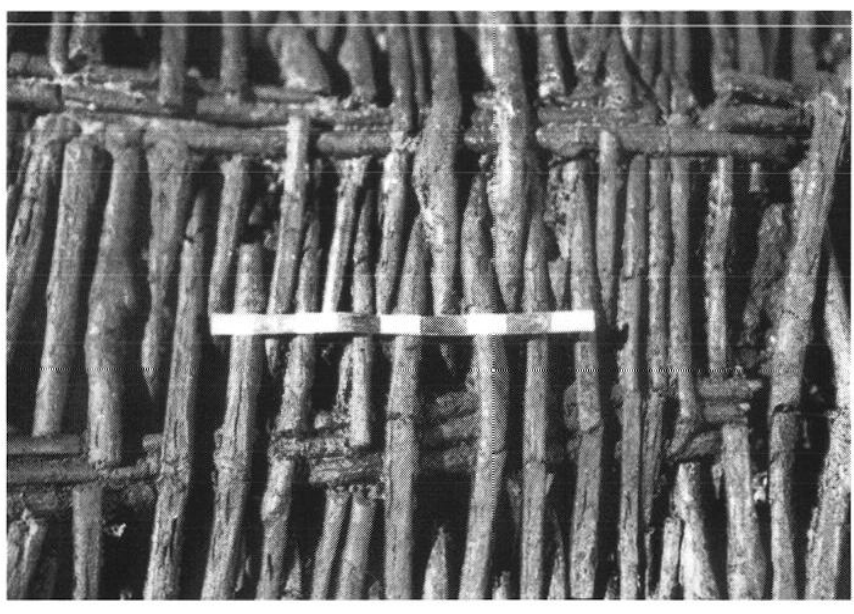

Fig. 7 - Délail du matériau de la nasse du Petit-Creusol (cliché N. Blanc ê E. Gury).

juger de l'ordre des étapes de sa fabrication, nous commençons sa description par celle du corps, bien visible, contrairement au goulet encastré à l'intérieur du corps et qui ne peut être observé que là où ce dernier a cédé, permettant ainsi d'appréhender sa structure ${ }^{16}$.

Les douze montants de la sortie (le douzième restitué) sont constitués de faisceaux de brins qui forment des fourches à une hauteur comprise entre 13 et $38 \mathrm{~cm}$, de sorte que l'entrée de la nasse comporte dix-huit montants.

16. En outre, la nasse est aujourd'hui présentée, après restauration, à l'envers de la position qu'elle occupait au moment de la découverte : ainsi se trouve masquée la déchirure qui permettait d'observer l'encastrement du goulet dans le corps.

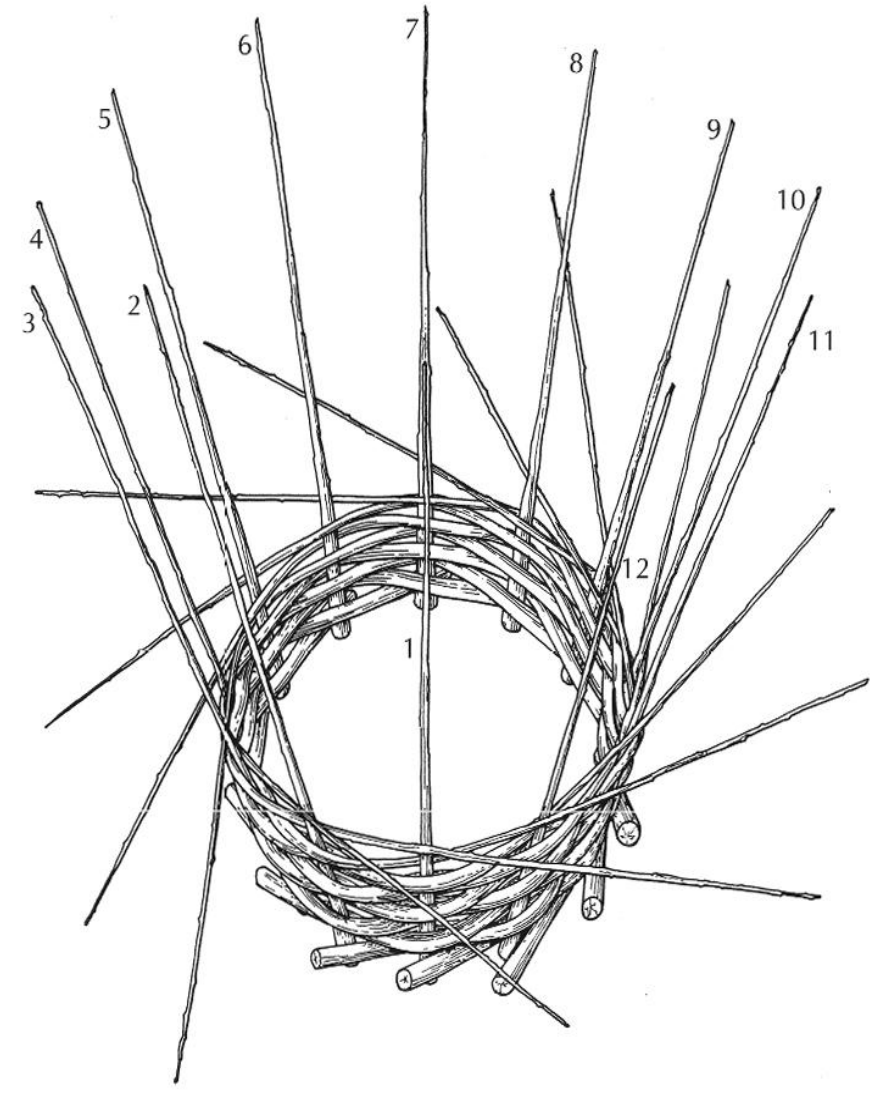

Fig. 8 - Mode de tressage du corps de la nasse du Petit-Creusot (dessin G. Monthel).

On remarque de gauche à droite, et de la sortie vers l'entrée (fig. 9 et 10$)$ ( $\mathrm{S}=$ sortie; $\mathrm{E}=$ entrée; $\mathrm{G}=$ goulet) :

- montant S 1, faisceau de trois brins (montant E 1) ; ce montant est réuni au suivant (S 2), à $6 \mathrm{~cm}$ de l'extrémité supposée de la nasse ;

- montant S 2, faisceau de quatre brins séparés en deux à $29 \mathrm{~cm}$ de hauteur; trois partent à droite ; le quatrième se voit adjoindre à gauche deux brins supplëmentaires qui l'encadrent à la fourche, de manière à former deux faisceaux de trois brins chacun (montants E 2 et E 3) ;

- montant S 3, faisceau de trois brins (montant E 4) ;

- montant $S 4$, faisceau de trois brins (montant E 5) ;

- montant S 5, faisceau de trois brins enrichi, à $15 \mathrm{~cm}$ de hauteur, de deux brins supplémentaires à gauche; le faisceau de cinq brins ainsi obtenu se divise à $38 \mathrm{~cm}$ de hauteur; un brin supplémentaire fourchu permet de constituer deux faisceaux de trois brins chacun (montants $\mathrm{E} 6$ et $\mathrm{E} 7$ ) ;

- montant S 6, faisceau de trois brins (montant E 8) ; 


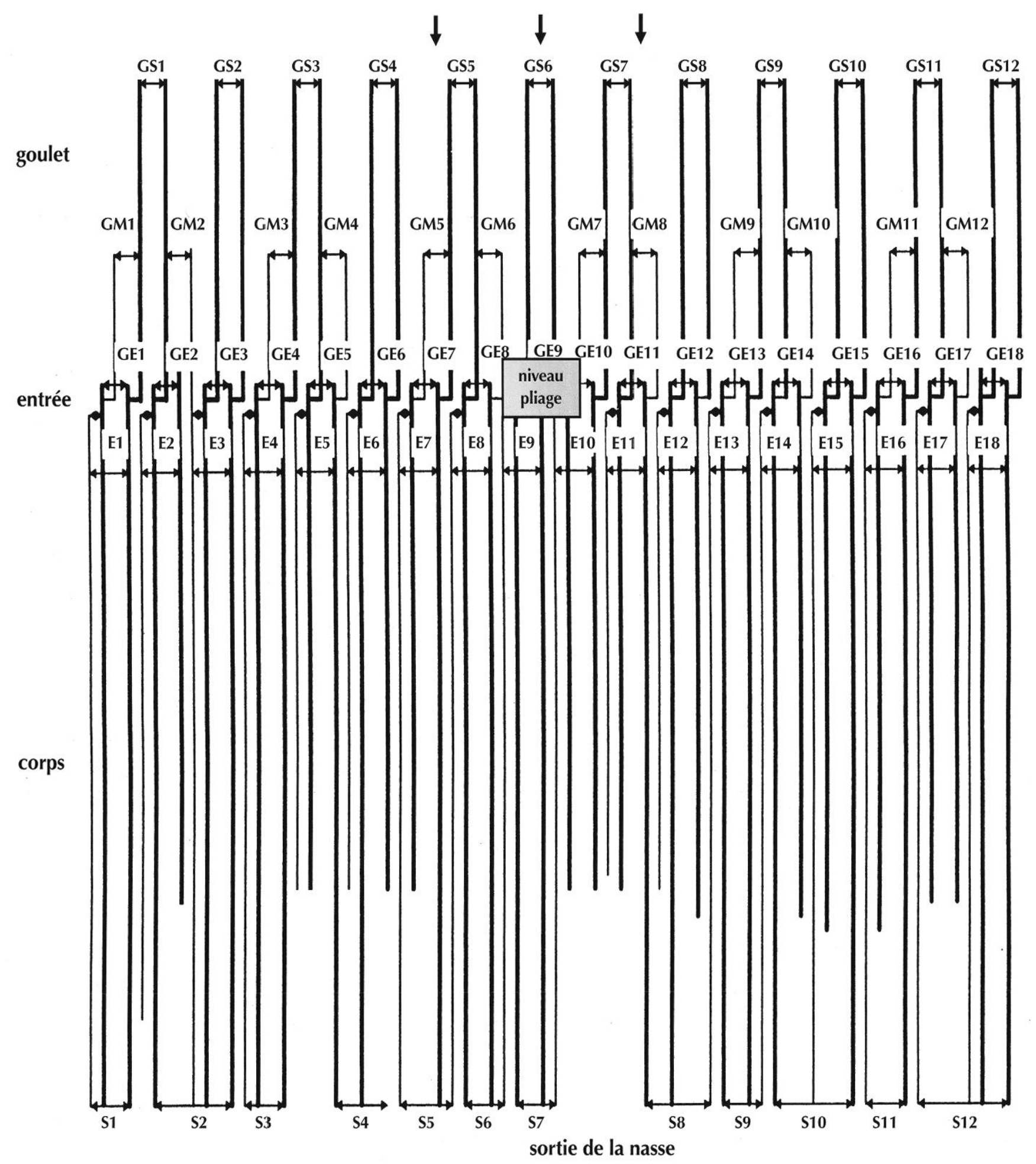

$\leftrightarrow$ ou $\bullet$ formation du faisceau

Fig. 9 - Analyse technologique de la construction de la nasse du Pelit-Creusot : GS, faisceaux de départ à la sortie du goulet (vers l’intérieur de la nasse) ; GM, nouveaux faisceaux obtenus par rajout de 6 brins; GE, faisceaux à l'entrée du goulet et au niveau de la pliure des brins pour former le corps; $E$, faisceaux à l'entrée du corps de la nasse avec le retournement des brins $(E=G E) ; S$, faisceaux à la sortie du corps de la nasse (dessin N. Blanc et F. Gury). 

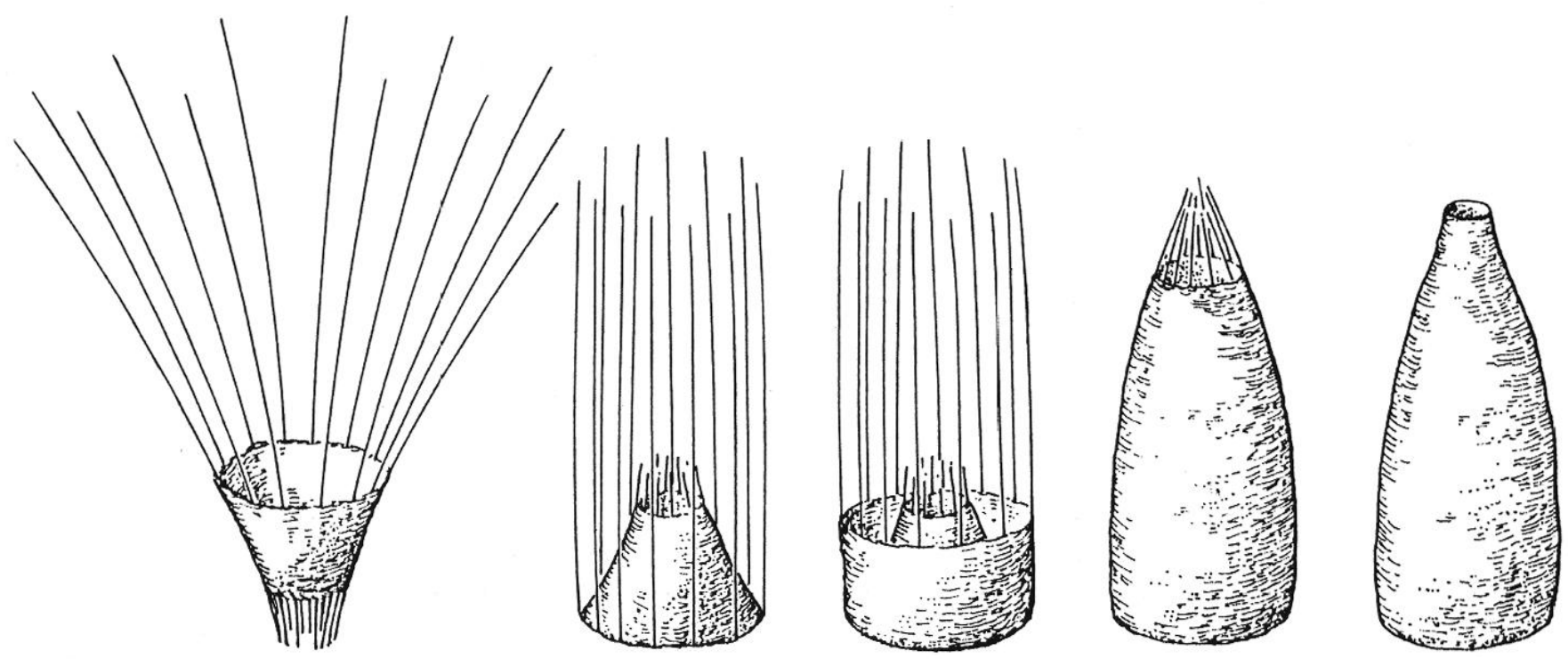

Fig. 10 - Schéma des étapes de la construction de la nasse du Petit-Creusot (dessin G. Monthel).

- montant S 7, faisceau de trois brins enrichi à $23 \mathrm{~cm}$ de hauteur d'un brin supplémentaire ; il se divise à $31 \mathrm{~cm}$ de hauteur ; les trois brins initiaux partent vers la gauche ; le brin supplémentaire part vers la droite, encadré par deux brins ajoutés pour former un faisceau de trois brins (montants E 9 et E 10 ) ;

- montant $\mathrm{S} 8$, faisceau de quatre brins se divisant à $15 \mathrm{~cm}$ de hauteur; trois brins partent à droite et le quatrième, à gauche, est encadré, à la fourche, par deux brins supplémentaires (formule identique à celle du montant S 2) (montants E 11 et E 12) ;

- montant S 9, faisceau de trois brins (montant E 13);

- montant S 10, faisceau de quatre brins se séparant en deux faisceaux de trois brins à $25 \mathrm{~cm}$ de hauteur grâce à l'ajout d'un brin de chaque côté (montants E 14 et E 15) ;

- montant S 11, faisceau de trois brins (montant E 16);

- montant S 12, faisceau de quatre brins se divisant à $30 \mathrm{~cm}$ de hauteur; trois brins partent à droite, le quatrième, à gauche, est enrichi de deux brins supplémentaires intérieurs à la fourche (montants E 17 et E 18).

\section{LE GOULET}

À l'entrée de la nasse, la jonction entre le goulet et les dix-huit faisceaux de brins qui constituent les montants du corps est endommagée (fig. 3 et 4 ). Il n'est donc plus possible d'observer la continuité de ces deux parties.

Nous ignorons également les dimensions exactes du cône : son extrémité pointue, engagée dans la substance de remplissage utilisée pour la restauration, n'est plus visible. Cependant la partie conservée qui mesure $44 \mathrm{~cm}$ de longueur doit être presque entière, car il ne manque réellement que la jointure du corps au goulet.

Sont visibles onze montants constitués de faisceaux de deux à trois brins d'une section comprise entre 3 et $6 \mathrm{~mm}$. Le large espace qui subsiste marquerait l'emplacement d'un douzième, détruit.

\section{LE MODE DE CONSTRUCTION}

La reconstitution du mode de construction de la nasse se heurte à deux difficultés. Du fait de la disparition de la jointure corps/goulet - qui nous prive d'un témoignage utile - la première difficulté concerne la liaison entre ces deux éléments; la seconde, qui découle de la première, tient à l'ordre des étapes de la construction. Sur ces deux points nous ne pouvons qu'avancer des hypothèses appuyées sur quelques indices.

Après un premier examen de la nasse restaurée, nous avons tout d'abord pensé que son corps avait été construit de la partie étroite (sortie) vers la partie large (entrée). À ce stade de l'étude, l'expérimentation menée 


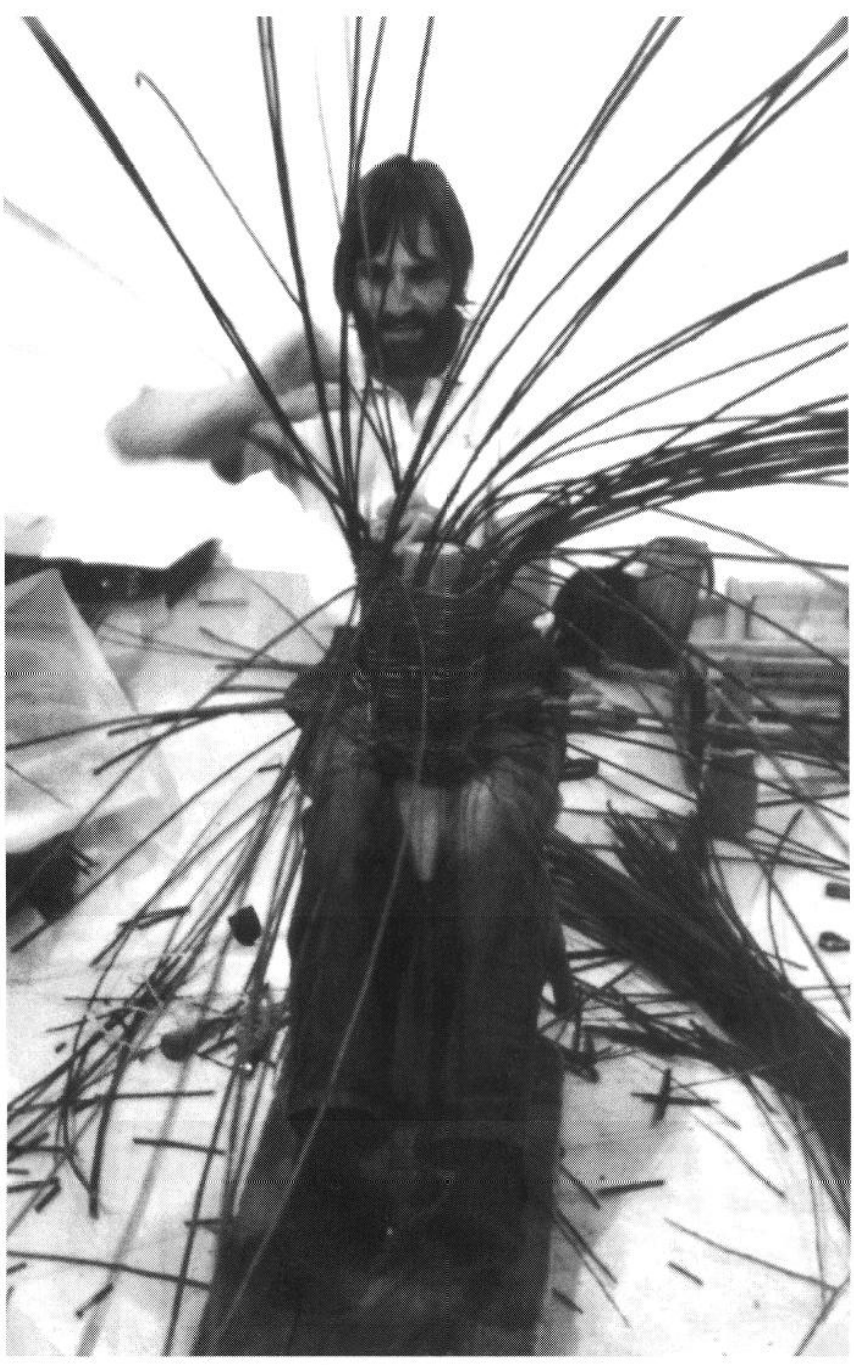

1

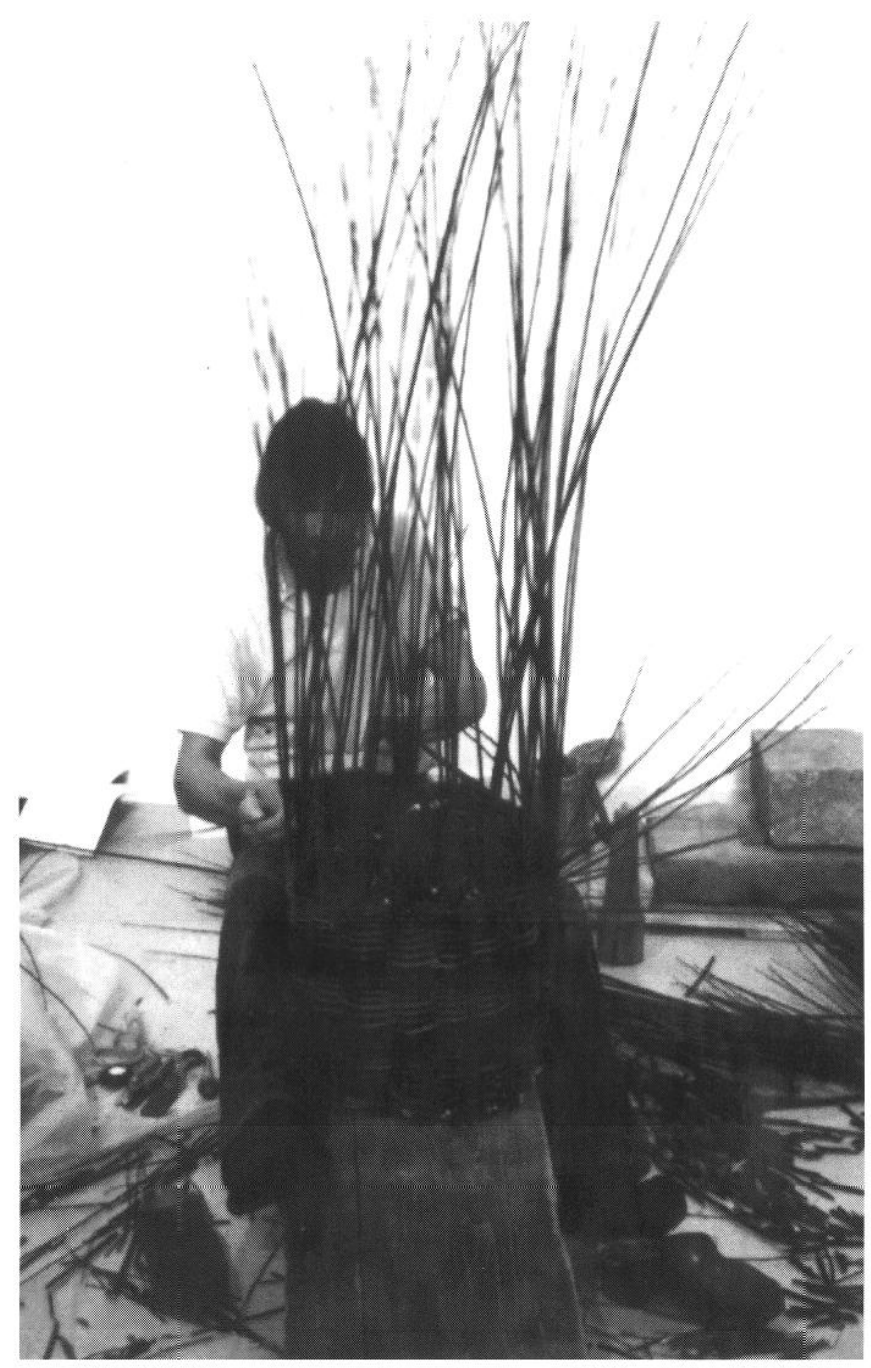

2

Fig. 11 - Reconstitution expérimentale de la nasse par G. Barbier :

1, première hypothèse (rejetée comme irréalisable);2, seconde hypothèse retenue (clichés $G$. Monthel).

par le vannier M. Guy Barbier ${ }^{17}$ sur la fiabilité de cette hypothèse l'a invalidée (fig. 11, $\mathrm{n}^{\circ} 1$ ). En effet, commencer le travail par la sortie de la nasse revient à utiliser en premier lieu l'extrémité fine des brins d'osier, ou cimes. L'artisan se trouve alors à manier une haute gerbe de tiges dont les extrémités épaisses, ou racines, pesant de tout leur poids, exercent une action centrifuge qui gêne considérablement, jusqu'à la rendre impossible,

17. G. Barbier (Les Brins d'Osier, 23, route nationale, F-36800 Le PontChrétien-Chabenet) s'est spécialisé dans la reconstitution des formes et des techniques anciennes, à partir des vestiges archéologiques, de l'iconographie et des témoignages ethnologiques. Il travaille en collaboration avec des archéologues et des musées français et étrangers (Barbier et al., 1999). la progression du tressage. Les impératifs techniques commandent donc que le corps de la nasse soit fabriqué de l'entrée vers la sortie en utilisant les brins de la racine à la cime (fig. $11, n^{\circ} 2$ ).

Le calibre de 3 à $6 \mathrm{~mm}$ des brins du goulet, l'irrégularité de leurs faisceaux de deux à trois brins, l'évidence d'une différence de structure entre le goulet - dont on repère encore 11 montants - et le corps de la nasse - qui présente vers l'entrée 18 montants - conduisent à supposer un tressage séparé des deux parties. C'est l'hypothèse de restitution retenue par Guy Barbier. Elle se fonde sur l'usage actuel, mais également sur l'observation, au niveau probable de l'entrée du goulet, de brins vrillés qui seraient les vestiges de l'attache torsionnée, 


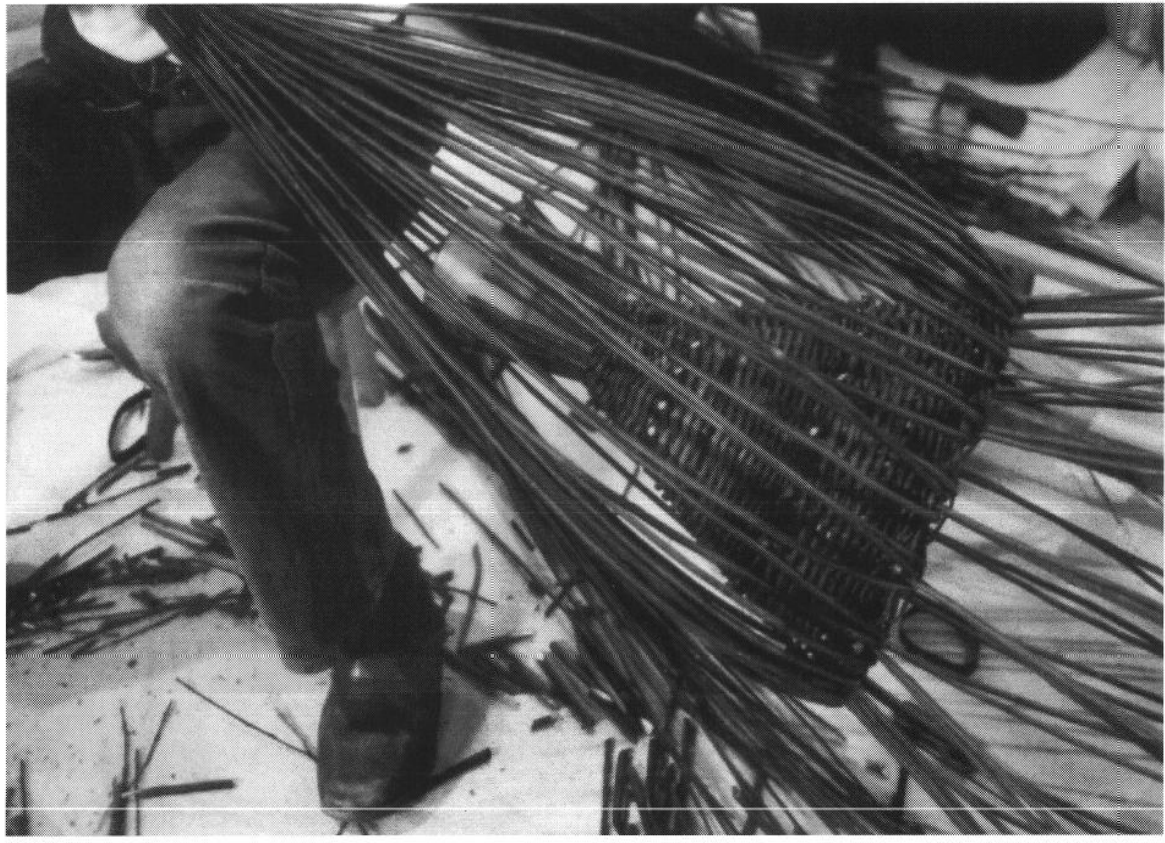

Fig. 12 - Reconstitution expérimenlale de la nasse par G. Barbier : enchaînement goulet-corps (cliché G. Monthel).

indispensable au maintien des brins du goulet, pliés et retournés pour permettre à la charpente du corps de la nasse de venir s'adapter. Le goulet aurait donc été d'abord construit, puis le corps de la nasse, en prenant appui sur lui, serait venu l'englober, de la partie large (entrée) vers la partie pointue (sortie) (fig. 10 et 12).

Selon cette hypothèse, le travail s'amorce avec la constitution de douze montants ou faisceaux de deux brins (GS 1 à GS 12), soit vingt-quatre brins (fig. 9). Au début du travail, ces brins initiaux sont maintenus par un anneau ou disposés autour d'un " crougnon " 18 (fig. 12). Après quelques centimètres de tressage (GM 1 à GM 12), un brin est ajouté à chaque faisceau de manière à augmenter le diamètre du goulet en forme d'entonnoir et obtenir, par regroupement des trente-six brins, dix-huit faisceaux ou montants au niveau de l'entrée du goulet (GE 1 à GE 18). Ces dix-huit montants sont alors pliés et couchés; c'est là que viennent se piquer les montants de la charpente du corps de la nasse (fig. 10 et 12). Cette pliure forme l'entrée de la nasse, aujourd'hui perdue.

18. En Dordogne, élément en bois de forme tronconique, dont le diamètre détermine celui de l'entrée du goulet de la nasse. En fait, n'importe quel objet grossièrement cylindrique peut en tenir lieu.
Une fois cette opération accomplie, on commence à construire le corps de la nasse à partir de dix-huit montants de deux brins (soit trente-six brins) piqués à quelques centimètres du bord du goulet. Chaque montant est ensuite, au niveau de la pliure, enrichi d'un brin. On obtient un total de cinquante-quatre brins réunis en dix-huit faisceaux de trois brins chacun (E 1 à E 18). Puis, progressivement, dans une zone comprise entre 40 et $15 \mathrm{~cm}$ de la sortie, certains brins sont abandonnés pour amenuiser la forme vers la sortie de nasse, qui ne compte plus pour finir que onze ${ }^{19}$ ou douze faisceaux de trois ou quatre brins chacun (S 1 à S 12). La diminution du diamètre du corps résulte de la fusion de douze des dix-huit faisceaux initiaux de manière à ramener leur nombre à onze (ou douze) à la sortie de la nasse. La nasse perd donc progressivement un tiers de ses montants.

Cette hypothèse de restitution de la nasse selon des principes de construction réguliers autour de nombres de brins multiples de 3 et de 12 est conforme à ce que nous sommes encore en mesure d'observer sur le corps de la nasse. Ce qui reste du goulet, en revanche, est loin de présenter la même régularité et coïncide moins bien avec la solution proposée par Guy Barbier. Rappelons que les onze montants visibles ou discernables du goulet sont constitués par des faisceaux de deux à trois brins d'une section comprise entre 3 et $6 \mathrm{~mm}$. Par ailleurs, ne sont plus visibles aujourd'hui que onze montants au maximum, sans qu'il soit possible, compte tenu de l'espace, de restituer plus d'un ou deux faisceaux à cet ensemble. Il faut admettre que l'entrée de la nasse et la partie supérieure du goulet sont arrachées sur une hauteur relativement importante puisque cet arrachement intervient en GM, c'est-à-dire au niveau de l'augmentation des brins et avant l'adjonction des six faisceaux supplémentaires (GM 1 à GM 12).

19. Si on compte pour un seul montant $\mathrm{S} 1$ et $\mathrm{S} 2$ réunis à $6 \mathrm{~cm}$ de l'extrémité supposée. 
Quoi qu'il en soit, la construction de la nasse, en apparence irrégulière, est profondément cohérente. Les dix-huit montants du départ, qui permettent d'obtenir douze montants à l'arrivée, comportent chacun trois brins. Il en résulte régularité et solidité du corps de la nasse. C'est à dessein que les montants, en fusionnant, forment à leur tour un nouveau faisceau de trois brins. Une épaisseur des montants trop disparate n'aurait, en effet, pas permis un tressage régulier; par ailleurs, le nombre de brins ne devait pas être trop élevé pour obtenir une ouverture suffisamment étroite. Rappelons qu'il s'agit de la sortie de la nasse, qui doit être bouchée ${ }^{20}$ pour empêcher le poisson de s'échapper. Ces faisceaux ne comportent donc que trois ou quatre brins et se voient adjoindre, au moment de la séparation, les brins nécessaires pour obtenir deux faisceaux réguliers de trois brins chacun. Le fait que cette opération ne s'effectue pas à la même hauteur et que les modalités en soient différentes vient de ce que le vannier antique a dû tirer le meilleur parti de brins très irréguliers, par leur taille sans doute et surtout leur épaisseur. Il obtient ainsi, à partir de brins non calibrés, un tressage qui semble parfaitement régulier et dont les « défauts » de construction ne sont discernables que par une analyse détaillée.

\section{LE TRESSAGE}

La clôture du corps est un travail de vannerie tissée du type armure toile, à partir de brins de 1,5 à $8 \mathrm{~mm}$ de diamètre (Balfet, 1952, p. 264-265, tabl. I, type 22) (fig. 6 à 8) ; en général, on peut les suivre en continu sur un quart de la circonférence. L'irrégularité du tressage vient en partie de celle du matériau, épais à la racine, effilé à la cime; certains brins peuvent être fourchus. Quelquefois le brin actif, faute d'un rameau entier suffisamment gros, est remplacé par un faisceau de deux, voire trois brins (fig. 6 et 9) ; ils sont quelquefois éclatés, ou même brisés à l'arête. Il est possible de compter une douzaine de ces bandes de tressage "en crocane ", d'un bel effet décoratif (fig. 5). Ces zones de travail, correspondant à l'utilisation d'un brin, sont, comme il se doit, de plus en plus hautes à mesure que diminue le diamètre de la nasse. La densité du tressage

20. Outre le tampon d'herbes évoqué plus haut, l'obturation de la nasse pouvait aussi bien être obtenue grâce à un bouchon de liège ou de bois, ou même un panier. n'est pas la même sur toute l'étendue de l'objet: de 25 brins aux $10 \mathrm{~cm}$ dans la partie étroite (ou sortie) de la nasse, elle monte jusqu'à $13-15$ brins aux $10 \mathrm{~cm}$ à l'autre extrémité (fig. 5). La restitution de Guy Barbier utilise pour la clôture du corps dix bandes de tressage " en crocane ", puis vers la sortie, sur les six derniers centimètres de la longueur restituée, une bande de tressage « à brins suivis ». Ce dernier mode de tressage destiné à consolider l'extrémité de l'ouvrage ne peut s'effectuer que sur un nombre impair de montants. Si l'artisan antique avait choisi cette option, invérifiable aujourd'hui vu la disparition des ultimes centimètres de la nasse, il aurait été contraint in extremis de réduire d'une unité le nombre des montants (S 1-S 12) afin de le ramener à onze et permettre cette réalisation.

\section{USAGE ET CONDITIONS DE PRODUCTION}

La pêche à la nasse en eau douce est bien attestée en Europe du Nord depuis le Mésolithique jusqu'à la Protohistoire, et les vestiges les plus anciens qui témoignent d'un épanouissement plutôt que d'une éclosion des techniques permettent de la faire remonter, avec une grande probabilité, au Paléolithique supérieur ${ }^{21}$. En Gaule, les premières nasses archéologiques connues sont celles de Noyen-sur-Seine (Seine-et-Marne), datées des années 7000-5000 avant J.-C. La mieux conservée, de forme conique, avec $1 \mathrm{~m}$ de hauteur maximum et $0,35 \mathrm{~m}$ de diamètre, est réalisée en clayonné à brins cordés, avec montants serrés et brins espacés ${ }^{22}$.

Paradoxalement, les trouvailles se font rares à l'époque historique et c'est la nasse celtique de Melz-surSeine (Seine-et-Marne), que nous avons déjà mentionnée à plusieurs reprises, qui reste le modèle le plus proche de la nasse du Petit-Creusot ${ }^{23}$.

21. Cf. Cleyet-Merle, 1989 ; une nasse de Nidlöse (Danemark) offre de nombreux points communs avec l'exemplaire de Melz-sur-Seine: technique du clayonné à brins cordés, montants serrés et brins espacés.

22. Cf. Mordant, 1987-1990, p. 28, fig. 12 et 1989, p. 121, notice 57 et fig. (avec bibliographie). Citons également un large fragment de $0,90 \mathrm{~m}$ sur $1,10 \mathrm{~m}$, de forme aplatie mais appartenant probablement à une nasse, trouvé au Gué des Piles (Chalon-sur-Saône) ; il est daté du Bronze final III (Gallia Informations, 1987-1988, 2, p. 39).

23. Le musée de Metz conserve des fragments de nasses gallo-romaines encore inédites et qui viennent d'être restaurées. Plus généralement, voir pour la Gaule le tableau des découvertes donné dans Thomasson, 1992, p. 94. 
L'iconographie offre des représentations de nasses, mais seulement pour la pêche en mer ${ }^{24}$, et aucun document ne provient de Gaule. Cette technique semble avoir été constante pendant toute l'Antiquité. Pour le monde grec, citons une coupe à figures rouges du peintre d'Ambrosios, datée de la dernière décennie du $\mathrm{VI}^{\mathrm{e}} \mathrm{s}$. avant J.-C., qui montre un jeune homme plongeant sa ligne dans une mer poissonneuse au fond de laquelle est posée une nasse de forme grossièrement quadrangulaire, qui rappelle nos modernes casiers à crustacés (Hölscher, 1992, p. 211-212, pl. 43,1). Dans le monde romain, ce sont les mosaïques d'Afrique du Nord les plus instructives : la mosaique d'Ulysse de Dougga ${ }^{25}$ et deux mosaiques de Soussc ${ }^{26}$ illustrcnt unc tcchnique particulière de pêche par chaîne de nasses (fig. 13). Elles sont liées ensemble par groupe de trois, tirées, posées ou relevées depuis un bateau; leur forme affecte celle d'une outre, avec corps ovoïde et étranglement à l'embouchure. Nous trouvons une confirmation de cette technique sur une mosaïque de Carthage ${ }^{27}$ ornée, en guise de bordure, de deux chaînes affrontées, formées respectivement de neuf et huit nasses ; elles ont sensiblement la même forme que les exemplaires précédents, et leur module $-0,44 \mathrm{~m}$ de long sur $0,25 \mathrm{~m}$ de large - reproduit sans doute, à défaut de dimensions exactes, des proportions réelles. Le motif se retrouve également sur la céramique africaine où des scènes de pêche montrent filets et nasses lancés ou tirés depuis des barques (Lissi-Caronna, 1995). Sur une mosaique de Rome de la fin du I ${ }^{\text {er }}$ s. avant J.-C., une vannerie de forme ovoïde qui semble fixée à un piquet dans un contexte marécageux, et d'où s'échappent des escargots, pourrait bien être une nasse abandonnée,

24. Les textes également ne nous renseignent que sur les nasses de mer; selon Pline, elles peuvent être tressées en jonc (Histoire naturelle, 21, 113); on les frottait avec des salaisons dont l'odcur ćtait ccnsćc attirer le poisson (Hist. nat., 9, 92), ou l'on y installait un appât (Hist. nat., 10, 194). L'expression est également employée comme en français de façon imagée pour désigner un piège : Plaute, Mil., 581 ; Cicéron, Correspondance, Att., 15, 20, 22 ; Silius Italicus, Guerres puniques, 5, 48.

25. Tunis, musée du Bardo, $\mathrm{n}^{\circ}$ inv. 2884 A (Poinssot, 1965, p. 224 fig. 18; Dunbabin, 1978, p. 257, n 8, pl. VIII 16 ; Blanc, Gury, 1990b, p. 202, fig. 4).

26. Musée de Sousse, $\mathrm{n}^{\circ}$ inv. 10.477 (Foucher, 1960, p. 45, $\mathrm{n}^{\circ} 57095$ [Sousse C5], pl. XXI ; Fradier, 1994, fig. p. 118) ; même schéma sur la seconde mosaïque, où un pêcheur debout relève la chaîne, tandis qu'un second, assis dans la barque, vide le contenu d'une autre nasse (n inv. 10.455) (Foucher, 1960, p. 91 n $^{\circ} 57204$ [Sousse B6], pl. XLVI).

27. Cf. Ennaiffer, 1973, p. 62, pl. 22 II ; pour plus de détails, voir : Bairem-Ben Osman, 1980, ı. 2, p. 182-198 nº 59, pl. 59.

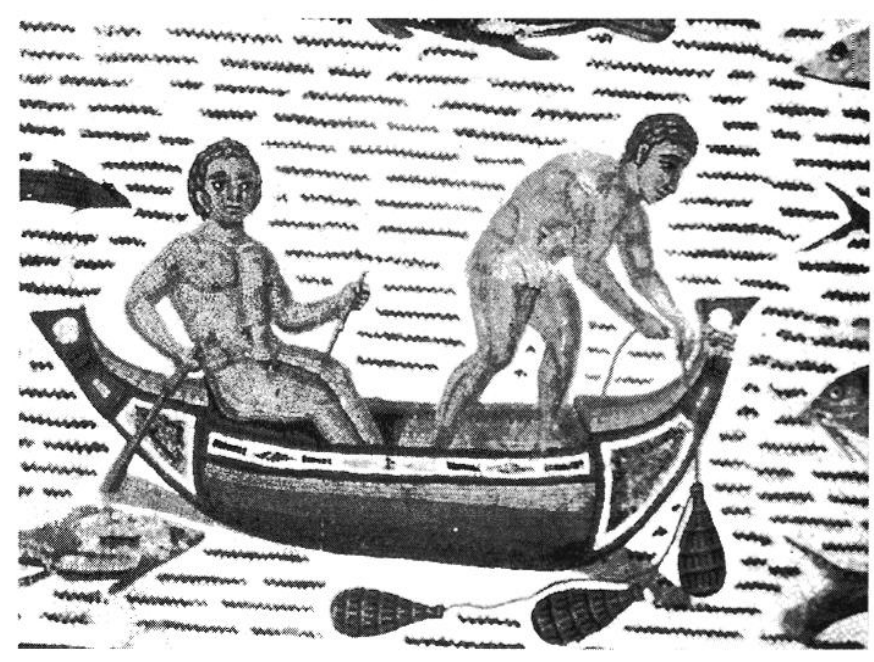

Fig. 13 - Mosaüque d'Afrique du Nord illustrant la pêche par chaîne de nasses (musée de Sousse, $n^{\circ}$ inv. 10 477) (photo Centre H. Stern de recherche sur la mosaïque).

plutôt qu'une corbeille ${ }^{28}$ (Gasparri, 1983, p. 674-676, pl. 102, 2) : elle est réalisée en cordé à montants serrés et brins espacés, tressage qui semble figuré sur les exemplaires africains de Dougga et Sousse, et qui est également celui des nasses mésolithiques de Noyen-sur-Seine.

L'iconographie ne nous apporte donc aucun éclaircissement sur la pêche en eau douce ${ }^{29}$. Comme l'ont montré les données de fouille, la nasse du Petit-Creusot était posée dans le lit de la rivière, dans le sens du courant, sans doute retenue au fond ou amarrée à la berge. De grande taille,

28. Sa forme tronconique haute et étroite la rapproche néanmoins de certaines corbeilles portées par des pêcheurs à la ligne qui semblent y conserver leur prise, sur des mosaiques d'Antioche : voir un Amour pêcheur de la maison de Ménandre (Levi, 1947, p. 199, pl. CLIXa) et un autre monté sur un dauphin de la maison de la barque des Psychés (Levi, 1947, p. 185, pl. XI.IC), cette vannerie serait utilisée à la fois pour attraper et transporter les poissons de petite taille (Levi, 1947, p. 185) ; cette nasse-corbeille se retrouve également sur une mosaíque de Carthage (Bairem-Ben Osman, 1980, t. 2, p. 240-248, n 83, pl. 83), et sur une autre de Dougga (Bouhlila, 1987, p. 127, pl. XXXVIIIc). Sur un exemplaire de Leptis Minus, conservé au musée de Lemta, une toilette de Vénus Anadyomène montre une barque avec un Amour pêcheur relevant ou posant une nasse de ce type, tandis qu'une seconde est suspendue à la poupe à un espar (Blanchard-Lemée et al., 1995, p. 150, fig. 108). Signalons enfin une mosaïque des environs de Taparura, près de Sfax, où un Amour porte, accrochée à un trident, une corbeillenasse du même genre, plutôt qu'un filet (Margerin, 1994, p. 443-450, $\mathrm{n}^{\circ} 52$, pl. XCIV haut)

29. Même silence des sources écrites; Ausone, au IVe s., évoque trois techniques de pêche dans la Moselle - à la ligne, au filet dérivant, au filet flottant - mais ne mentionne pas la nasse (Mosella, 240-258). 


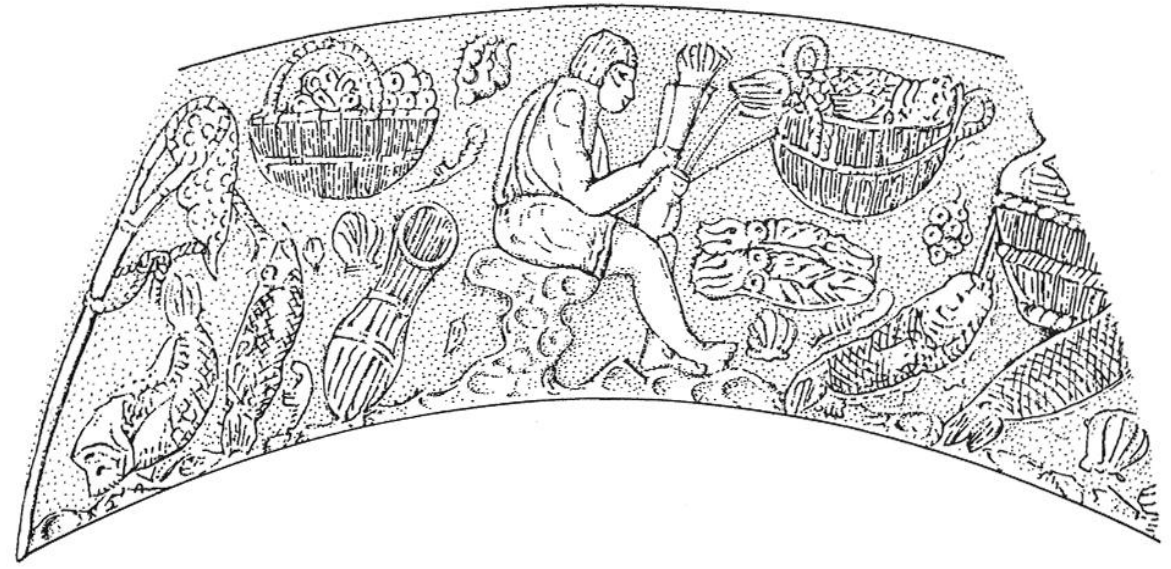

Fig. 14 - Frise de l'œnochoé d'Eyged, Hongrie (dessin G. Monthel d'après Linfert, 1974).

elle ne semble pas conçue pour un poisson particulier, comme l'anguille, mais pouvait sûrement piéger la plupart des espèces courantes dans la Saône : menu fretin ou plus gros gibier, comme tanches, brèmes, brochets ${ }^{30}$, etc.

Conçue et réalisée avec habileté, elle témoigne d'un savoir technique : c'est l'œuvre d'un spécialiste. Il s'agit en effet d'un outil et sa construction obéit à des règles qui sont garantes de son efficacité. S'agit-il pour autant d'un travail artisanal, attestant la présence d'un centre de production dans la région ${ }^{31}$ ? Rien n'est moins sûr car sa qualité n'exclut pas qu'elle ait été réalisée par son utilisateur, le pêcheur lui-même. Une œnochoé de bronze, provenant d'Eyged près de Budapest et datée du règne de Néron, permet au moins de le supposer (fig. 14). On y voit, sur un rivage rocheux, un pêcheur environné de poissons et de corbeilles, occupé à réaliser une nasse d'un modèle identique à celle figurée derrière lui. Il a déjà confectionné la moitié inférieure, de forme ovoïde, et dépassé le goulet d'étranglement: sur les montants écartés, il s'apprête à tresser la partie supérieure, sorte de cylindre évasé qui constitue l'ouverture du piège ${ }^{32}$. Une

30. Notons que l'anguille et le brochet étaient dominants dans l'ichtyofaune associée à la nasse de Noyen-sur-Seine (Mordant, 1987-1990, p. 27).

31. Sur les lieux et conditions de production de la vannerie, voir Mingaud, 1992. Une étude récente (Monacchi, 1996) vient ajouter un nouveau témoignage - une urne funéraire d'Ameria (Italie) - au bref corpus des artisans vanniers attestés par les inscriptions ou les outils figurés sur leur sépulture.

32. Cf. Gaitzsch, 1986, p. 88, n 32 et fig. ; Linfert, 1974, p. 17 et p. 22, pl. 15, 2, fig. 2. Voir aussi une nasse d'une forme proche, avec étranglcment du corps au nivcau du goulct, sur un plat de Lovere (Italie), daté de la première moitié du II's. (Linfert, 1974, p. 26; pl. 17, 3). scène du même type est évoquée dans un passage des Guerres puniques (5, 47-52) de Silius Italicus où Hannibal préparant un traquenard à l'ennemi est comparé au pêcheur tressant le goulet de sa nasse : " [...] l'habile pêcheur, tressant en osier une nasse légère à l'ouverture béante, entrelace avec un soin particulier les brins à l'intérieur : il les rapproche progressivement en formant pointe vers le centre de la coque où il fixe les cimes en les resserrant et, grâce au piège formé par cet étranglement, il interdit au poisson qu'il a tiré de l'onde, attiré par une entrée facile, de revenir en arrière. ${ }^{33}$.

\section{LA CORBEILLE}

Il s'agit d'une corbeille basse dont le fond n'est pas distinct de la clôture (fig. 15).

Diamètre le plus grand conservé : $62 \mathrm{~cm}$; diamètre restitué possible : $72 \mathrm{~cm}$ ?

\section{MATÉRIAU}

Sa réalisation met en œuvre des matériaux variés, non écorcés, présentant de nombreuses fourches, des nœuds, des brins tordus, des branches et des brindilles (fig. 17).

33. Traduction N. Blanc. Notons que Silius Italicus décrit le tressage du goulet comme postérieur à celui du corps, ce qui est matériellement impossible, sans doute parce que l'image est destinée à illustrer le mécanisme du piège. À moins qu'il nc se réfère à un type différent de construction en deux parties, tressées séparément, puis emboîtées et fixées. 
L'analyse effectuée selon la même technique que la nasse mais à partir de six échantillons révèle une composition non homogène.

Les montants sont en saule (Salix sp.) ; la disposition alterne des attaches et les distances entre les bourgeons correspondraient peut-être au saule à oreillettes (Salix aurita L.) ou au saule cassant (Salix fragilis L.).

Le remplissage comporte également du saule, de même variété que pour les montants, mais s'y ajoute de la clématite (Clematis vitalba L.), identifiée par la configuration anatomique des rameaux, avec les départs réguliers et couchés des pédoncules doubles, supportant les brindilles à feuilles (fig. 17, $n^{\circ}$ 2). Selon l'auteur de l'analyse, ces lianes, dangereuses car vénéneuses, ont été utilisées en vannerie grossière, ou pour faire des liens, mais presque jamais pour des travaux fins ou de commerce.

Le remplissage de la clôture comportait également du cornouiller (Cornus sp.); ce peut être du cornouiller mâle (Cornus mas L.) ou du cornouiller sanguin (Cornus sanguinea $\left.L_{\text {. }}\right)$, tous deux présents dans la région. Le cornouiller sanguin est le plus apte à être vanné, mais il serait attesté pour les montants plutôt que pour la clôture, ce qui n'est pas le cas ici.

L'hétérogénéité du matériau se reconnaît dans l'irrégularité de la densité du tressage (fig. $17, \mathrm{n}^{\circ} 1$ ). Le diamètre des brins actifs est compris entre $1,5 \mathrm{~mm}$ et $9,5 \mathrm{~mm}$ et celui des montants entre $6 \mathrm{~mm}$ et $9 \mathrm{~mm}$.

\section{CONSTRUCTION}

Comme la nasse, la corbeille entre dans la catégorie des vanneries clayonnées à montants parallèles. Mais les conditions de conservation rendent difficile une description technique précise de l'objet. De nombreux éléments semblent avoir bougé ou manquent. Sur un rayon de $15 \mathrm{~cm}$, le remplissage a disparu complètement et les montants superposés au centre sont désarticulés, désunis, déplacés. Une partie de ces manques sont dus aux manipulations de la restauration. Néanmoins, au moment de la découverte, la clôture du fond était déjà absente et son état de détérioration explique qu'elle ait été abandonnée, retournée, dans un contexte de dépotoir (fig. 15).

On peut cependant restituer une construction sur fond rayonnant superposé (fig. 16) : quatre faisceaux de quatre brins chacun donnent, après division, seize

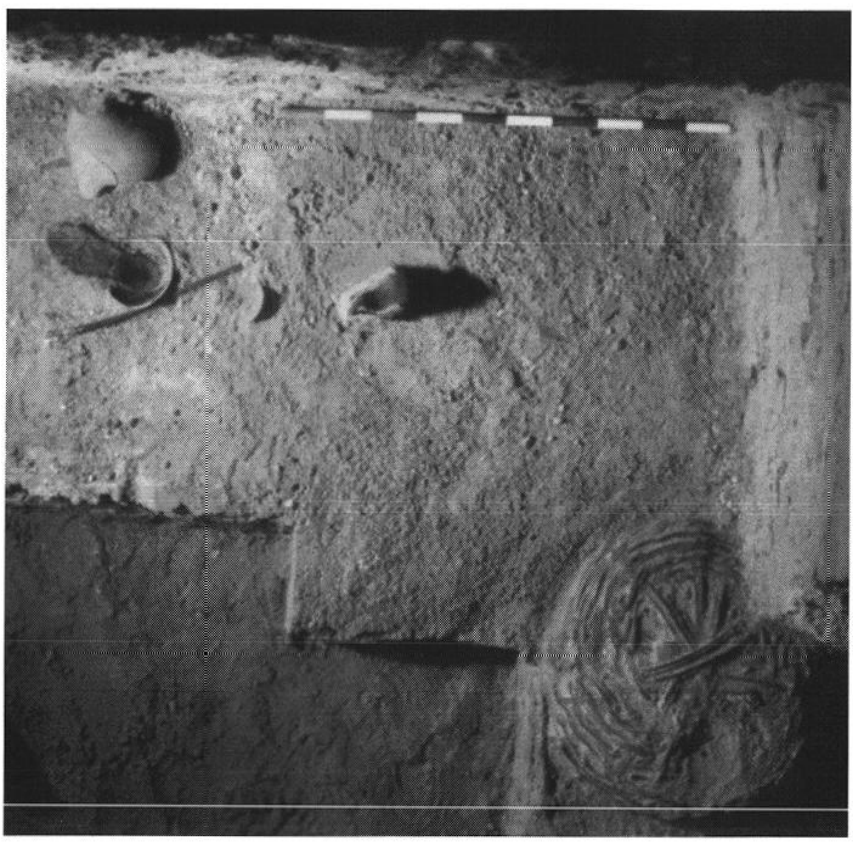

Fig. 15 - La corbeille du Petit-Creusot (photo de fouille, cliché (. Monthel).

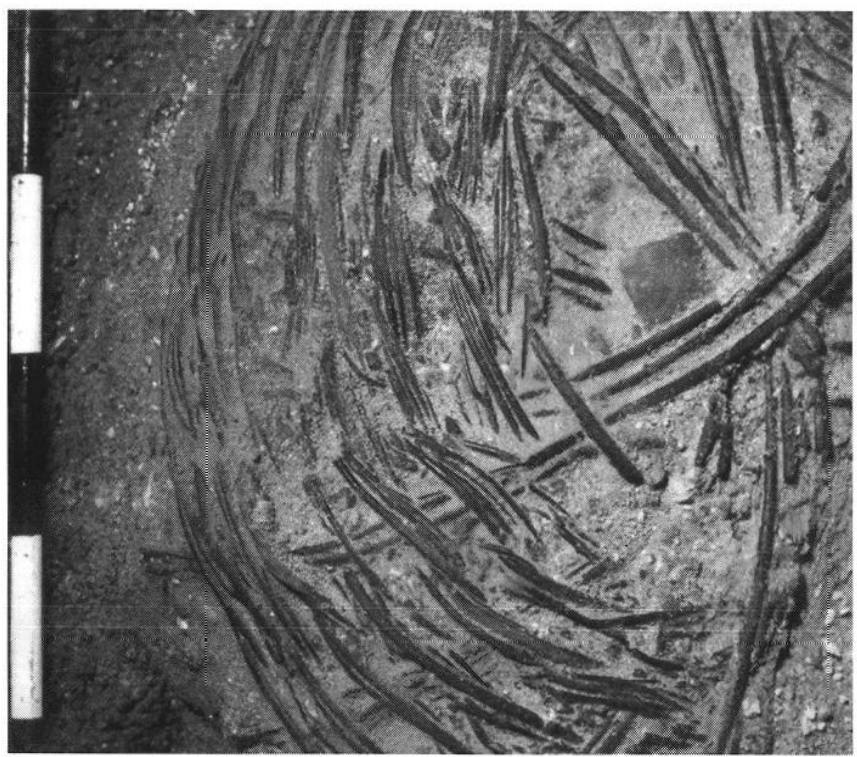

Fig. 16 - Détail de la corbeille du Petit-Creusot (photo de fouille, cliché G. Monthel).

montants et, après adjonction de nouveaux brins, un nombre difficile à apprécier de montants supplémentaires. Treize sont conservés. D'un diamètre compris entre $9 \mathrm{~mm}$ et $6 \mathrm{~mm}$, ils se présentent en général par faisceau de deux brins, mais sans exclusive. 


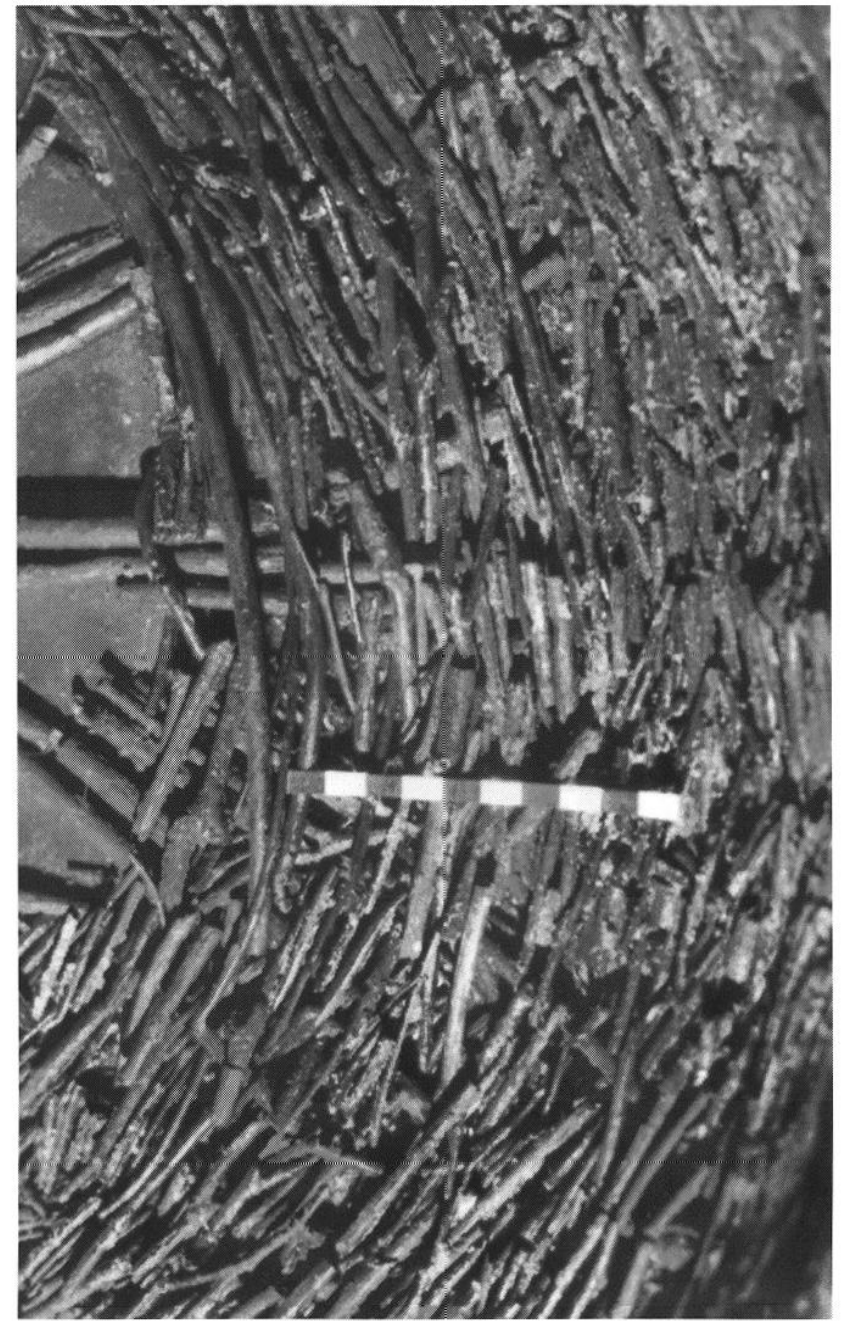

1

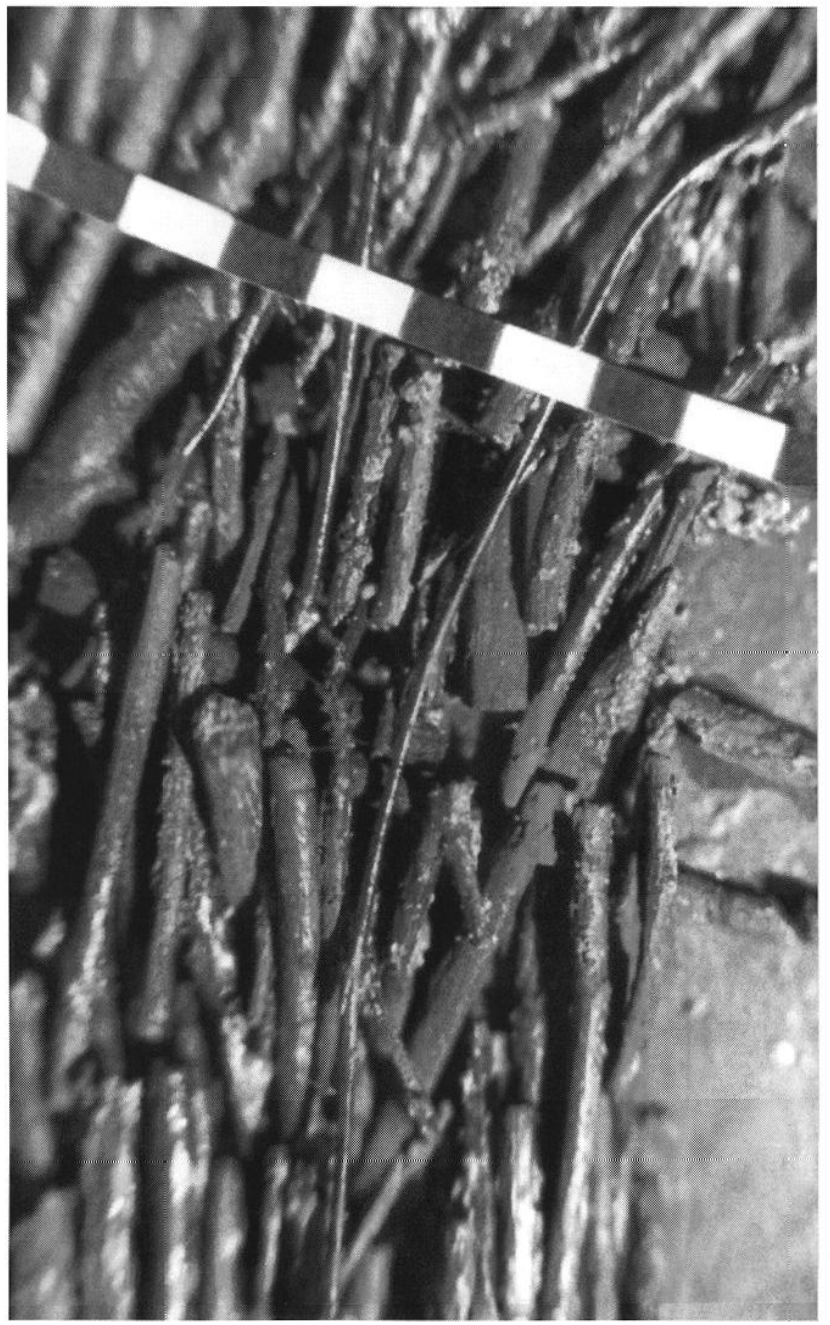

2

Fig. 17 - Détails du tressage de la corbeille du Petit-Creusot (clichés N. Blanc et F. Gury).

Le remplissage est un grossier clayonnage de brins, branches et brindilles dont le diamètre varie entre $9 / 10 \mathrm{~mm}$ et $1 / 1,5 \mathrm{~mm}$, avec une moyenne de $4 \mathrm{~mm}$ (fig. 17). Ces éléments peuvent être utilisès en mèches de deux à six brins et plus. L'état de conservation de l'objet ne permet pas de se faire une idée de la longueur des brins actifs. Si dans l'ensemble ils ont un aspect très segmenté, certains ont pu avoir jusqu'à $23 / 24 \mathrm{~cm}$. Le résultat est relativement épais ; la cohésion de l'ensemble est obtenue par l'entremêlement des nappes avec, de place en place, de gros brins ; les fourches contribuent à densifier le réseau des entrelacements (fig. 17). La densité de la nappe est très irrégulière: des mesures effectuées en trois endroits différents donnent, à titre d'exemple, 16, 19 et 24 brins pour $10 \mathrm{~cm}$.
On note l'absence de torche, cordon qui termine le fond et où sont fixés les montants verticaux; c'est l'indice d'une forme basse. Il est impossible de savoir comment était faite la bordure, et de déterminer si la corbeille était pourvue d'un moyen de préhension.

\section{USAGE ET CONDITION DE PRODUCTION}

Bien que la corbeille ait été retrouvée en partie écrasée, ce qui interdit une description très précise de sa forme, elle entre vraisemblablement dans la catégorie des corbeilles circulaires plates, bien connue par les monuments figurés. Ce sont en général des formes 
employées pour la présentation des mets (fruits, pain, etc.) : elles sont placées sur les genoux d'Epona ou des déesses-mères, emplies d'offrandes, dans les reliefs cultuels ou funéraires de la Gaule du Centre et du Nord ${ }^{34}$. La corbeille d'osier trouvée dans la tombe d'une jeune femme aux Martres-de-Veyre (Puy-de-Dôme) ${ }^{35}$, datée des $\mathrm{I}^{\mathrm{er}}-\mathrm{II}^{\mathrm{e}}$ s., quoique plus petite (diamètre: $0,26 \mathrm{~m}$ ), est proche par sa forme de l'exemplaire de Chalon; mais sa réalisation est plus soignée: c'est une vannerie fine, très légère, ornée sur le pourtour d'un élégant croisillon ; elle contenait des fruits, ce qui devait être son emploi habituel, rien ne permettant de supposer des formes particulières pour les usages funéraires et religieux.

Le caractère rustique de la corbeille de Chalon semble l'exclure du service d'une table raffinée; sa forme basse n'est guère adaptée au transport ou au stockage. Trouvée dans le même contexte que la nasse, il est tentant de la faire entrer dans le matériel de pêche. Aurait-elle appartenu à un pêcheur qui, commercialisant lui-même directement le produit de son travail, y aurait exposé sa marchandise ? Notons que certaines corbeilles qui environnent le pêcheur de l'œnochoé de Cologne présentent une forme semblable, agrémentée de deux petites anses 36 (fig. 14). Mais ce n'est évidemment qu'une hypothèse puisque aucune trace du contenu n'a pu être identifiée.

L'iconographie nous fait connaître divers types de corbeilles à poissons, plus ou moins profondes, souvent en vannerie souple et pourvues d'un moyen de préhension, anse ou poignées. Mais là encore ce sont les mosaïques africaines ${ }^{37}$, auxquelles s'ajoutent quelques

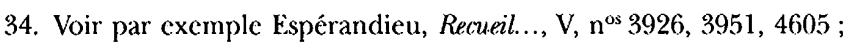
VIII, $\mathrm{n}^{\text {os }} 6247,6307,6336,6344,6356,6457 ; \mathrm{XI}, \mathrm{n}^{\text {os }} 7736,7767$, etc.

35. Cf. Audollent, 1923, p. $307 \mathrm{n}^{\circ} 34$. pl. VII ; diamètre : $26 \mathrm{~cm}$; hauteur : $4 \mathrm{~cm}$.

36. Cf. I infert, 1974, fig. 2. I.es collections du musée national des Arts et Traditions populaires comportent de nombreuses formes circulaires basses, servant à la présentation du pain et autres denrées (Jaoul, Goldstein, 1990, $\mathrm{n}^{\circ} 552,572$ ), mais souvent aussi d'usage inconnu $\left(\mathrm{n}^{\mathrm{os}} 573,577,605-606,612\right)$. Citons également un exemplaire provenant de Corse, bordé de liège et servant à contenir du matériel de pêche, lignes, hameçons, etc. (Jaoul, Goldstein, 1990, n 562).

37. Villa de Dar Buc Ammera à Zliten : large, basse, à anses souples (Aurigemma, 1960, p. 55-60, pl. 172) ; Sousse, même type mais plus haute (Foucher, 1960, p. 117, n ${ }^{\circ} 57.260$, pl. LXIV); Leptis Magna, maison d'Orphée : forme rigide, plutôt haute, à anses (Aurigemma, 1960, p. 52-54, pl. 107 et 111).

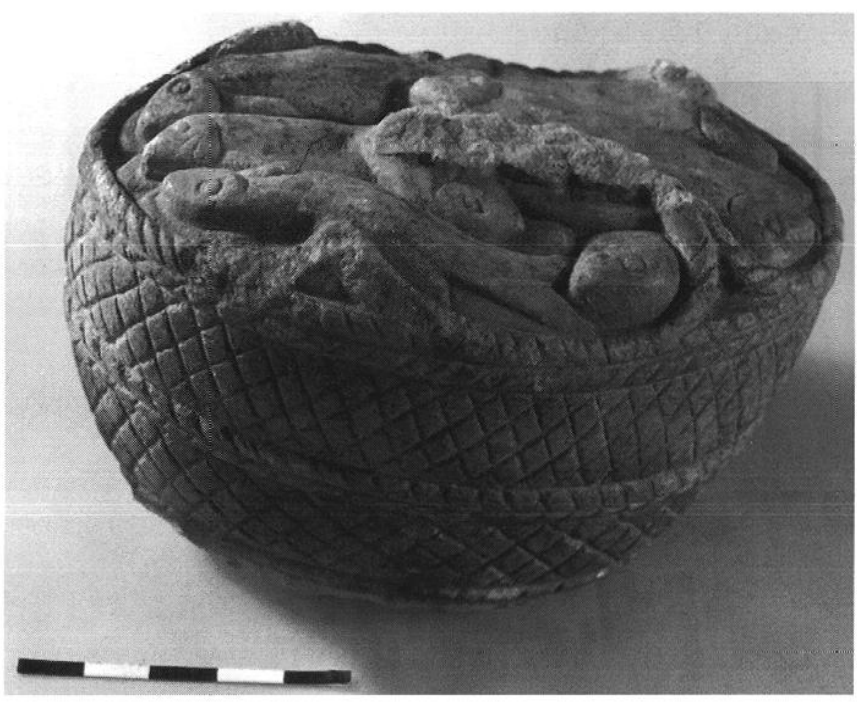

Fig. 18 - Corbeille d'Orbe-Boscéaz, Berne,

Bernisches Historisches Museum (cliché Bern. IIist. Museum).

natures mortes campaniennes ${ }^{38}$, qui fournissent l'essentiel de la documentation. Pour la Gaule, outre le pêcheur de Lyon qui porte un petit panier à deux anses ${ }^{39}$, on citera une ronde-bosse de pierre figurant une corbeille de forme plutôt basse, emplie de poissons, conservée au musée historique de Berne ${ }^{40}$ (fig. 18) ; le profil tronconique convexe asse $z$ mou suggère une texture plutôt souple, ce que confirme le tressage, rendu par des croisillons qui évoquent peut-être un travail à jour; un cordon à mi-hauteur et une bordure soignée dans laquelle sont prises deux petites poignées latérales complètent le modèle qui n'est pas sans rappeler une autre corbeille de poissons qui ornait le médaillon

38. Herculanum : couffin souple à deux anses molles (Maiuri, 1958, p. 44-45, fig. 19) ; voir aussi les exemples rassemblès dans ('roisille, 1965 , p. $81, \mathrm{n}^{\circ} 206$, pl. 36,72 (même type) ; p. $71 \mathrm{n}^{\circ} 163$, pl. 37,73 (même type) ; p. $37, \mathrm{n}^{\circ} 37, \mathrm{pl} .42,83$ (à anse unique). Forme circulairc basse, rigide, à anses souples : Pompéi IX 12 [pièce n] (Varone, 1993, p. 629-630, pl. CLX, 2).

39. Cf. Espérandieu, Recueil..., III, n 1769 : il semble réalisé dans la technique du spiralé (faisceau de fibres enroulé sur lui-même et cousu) et s'apparente à un couffin.

40. $\mathrm{N}^{\circ}$ inv. BHM 14789 (diam. 0,12 m) ; elle a été mise au jour le 15 septembre 1749 dans l'ancien cimetière gallo-romain de la villa d'OrbeBoscéaz: Reoue historique vaudoise, 1929, p. 339-342 ; elle est illustrée dans Bandi, 1968, p. 514, fig. 4. Nous remercions ici le musćc historiquc de Berne qui nous en a aimablement fourni une photographie. Les cassures suggèrent une restitution proche de la corbeille de I yon, citée note 38 . 
central d'un plat d'argent du trésor de Graincourt-lèsHavrincourt (Pas-de-Calais) ${ }^{41}$ sans que l'on puisse dire si c'est le reflet d'une réalité ou la simple reproduction d'un motif décoratif.

À la différence de la nasse, cette corbeille, qui met en ouvre de manière assez grossière le tout venant en terme de brindilles et de rameaux végétaux facilement disponibles, révèle un travail d'amateur. C'est pourquoi, on peut hésiter à lui attribuer la même origine. Toutefois, n'étant pas un véritable instrument, la qualité d'exécution n'avait pas la même importance que pour la nasse.

En tout cas, il est quasi certain qu'elle n'a pas été acquise dans le commerce, mais produite par l'utilisateur lui-même, comme c'était souvent le cas; cette pratique est bien attestée dans le cadre de la villa, où les agronomes recommandent d'utiliser la mauvaise saison pour produire les ustensiles nécessaires au travail agricole ${ }^{42}$.

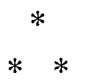

Ces deux documents apportent une contribution capitale à l'histoire de la vannerie en Gaule romaine jusqu'à maintenant pauvre en vestiges. Ces derniers, peu nombreux et le plus souvent fragmentaires, nous rendaient tributaires des textes et des représentations figurées pour cerner les matériaux, les formes et les techniques. L'identification de deux variétés adventices, Salix purpurea et Salix fragilis ou aurata viennent éclairer le passage peu explicite de Pline ${ }^{43}$ consacré aux différentes catégories d'osier. L'emploi du cornouiller sanguin, supposé à partir d'un vers de Virgile ${ }^{44}$, et de la clématite, déjà reconnu mais sans possibilité de vérification, sur des corbeilles exhumées en Vendée au siècle

41. Cf. Baratte, Painter (éds), 1989, p. 138-140 n 87, fig. Il est caractéristique de la production des ateliers de Gaule au III' $\mathrm{s}$. après J.-C.

42. Cf. Blanc, Gury, 1989, p. 200 et 1990a, p. 32 ; Mingaud, 1992. Les textes sont confirmés par le célèbre calendrier de Saint-Romain-en-Gal qui illustre cette activité dans le tableau consacré aux travaux des mois de janvier-février (Lancha, 1981, p. 213-214, pl. CXIVa).

43. Cf. Pline, Hisl. nat., 16, 174-177; voir le commentaire du livre XVI de J. André, collection des Universités de France, Paris, 1962, p. 157-158; Blanc, Gury, 1989, p. 199 et 1990a, p. 33.

44. Georg. I, 266 : Nunc facilis rubea texalur fiscina verga; cette interprétation est proposée dans André, 1985, p. 273, s.v. virga rubea. dernier (Baudry, Ballereau, 1873, p. 129 et 311, fig. 1) et aujourd'hui disparues, se trouve confirmé. On note l'emploi d'osier vert non écorcé pour la vannerie commune, ainsi que la tendance à utiliser sans discrimination toutes les ressources du terroir. Nous n'insisterons pas sur l'apport que constituent ces objets pour notre connaissance tant de la pêche en rivière que de l'environnement paléobotanique des rives de la Saône au $\mathrm{III}^{\mathrm{e}} \mathrm{s}$. ; mais nous retiendrons que c'est le premier témoin bien conservé de nasse pour la Gaule romaine ${ }^{45}$, contribution non négligeable à l'histoire des techniques et au corpus de l'instrumentum en vannerie. Le caractère très périssable du matériau ne doit pas faire oublier que la vannerie (employée pour les ustensiles de cueillette, de stockage, de transport, le clissage et paillage du verre et des amphores, le mobilier, les caisses de char, les outils du pressoir comme filtres, passoires, etc.) était omniprésente dans la vie quotidienne.

Enfin, les vestiges découverts au cours de la fouille confirment l'implantation du port antique au sud de la ville actuelle ${ }^{46}$, non plus seulement sur la rive droite de la Saône, voire sur des îles au centre de son lit, mais aussi sur sa rive gauche, situation jusqu'alors controversée. De plus, ils fournissent des éléments nouveaux sur le faciès de la rivière aux II ${ }^{c}$ et III ${ }^{c}$ s. après J.-C.

45. Sur les vestiges archéologiques actuellement recensés, voir Thomasson, 1992, p. 86-87.

46. Il est en effet judicieux de supposer que la plus grande part de la navigation qui venait du sud, où le négoce était particulièrement florissant, s'arrêtait au sud de la ville actuelle (comme c'était encore le cas au début du XIX"s. au port Guillot dit "le Porto" qui était « la dernière étape des hâleurs de la Saône entre Lyon et Chalon "; on y trouvait auberges et écuries : $c f$. Anonyme, 1812a et b ; Chevrier, 1883), au point de rupture de charge, mais aussi dans la zone où le tracé aval de la rivière, d'axe nord-sud, s'infléchit en amont vers l'est. Pourquoi les denrées destinées à d'autres directions que celle du nord-est auraient-elles été conduites de ce côté, alors qu'à partir de ce lieu la géographie n'offre plus aucun obstacle d'importance empêchant d'utiliser les nombreuses voies terrestres? Les découvertes archéologiques des $\mathrm{XIX}^{\mathrm{e}}$ et $\mathrm{XX}^{\mathrm{e}} \mathrm{s}$. viennent confirmer la localisation au sud de Chalon de ce port qui, dans l'Antiquité, pouvait s'ćtendrc loin en aval de la ville actuelle. Récemment encore, en 1975, lors du creusement, à partir de la rive gauche, de la darse de la zone industrielle sud, sur la commune de Saint-Marcel, deux " constructions en pierre ", perpendiculaires à la rivière, longues d'une cinquantaine de mètres et larges de vingt, "pleines " de céramique, ont été détruites par les engins (informations orales des ouvriers). Sans doute fallait-il voir ici la présence d'éventuels horrea, à environ $3,5 \mathrm{~km}$ de l'actuel centre ville. l.e développement économique et stratégique de Chalon doit sans doute autant à sa situation, au débouché et au centre des grandes ouvertures des bassins qui l'entourent, qu'à sa rivière. 
Pour orienter son cours suivant l'axe nord-sud, la rivière décrit un large méandre, immédiatement au sud du site actuel de Chalon (fig. 1). Or, qui dit méandre dit obligatoirement, dans certaines conditions physiques, déplacement du cours d'eau, quel qu'il soit. Dès lors, la Saône, à cet endroit, cesse d'être cette " paisible et indolente rivière ». Elle tend à ronger, à éroder sa rive droite concave, tout en fuyant, par des dépôts d'alluvions, sur sa rive gauche convexe. Elle se déplace ainsi de l'est vers l'ouest (fig. 19). Ce phénomène est encore sensible à notre époque puisqu'il n'est pas rare, lors de crues d'importance ordinaire, que la Saône détruise des portions du perré qui protège la rive droite de ce méandre, tandis que, sur la rive gauche, le bras qui longe l'île dite de La Benne-la-Faux, située en aval du pont des Dombes, s'ensable d'année en année ${ }^{47}$. C'est ce mouvement avec dépôts de sédiments sur la rive gauche de la rivière qui est désormais prouvé par les vestiges du Petit-Creusot.

Ils jettent également un jour nouveau sur des découvertes du siècle dernier dans la même zone, là où deux îles s'allongent à l'est du chenal actuel de la Saône, en aval du pont des Dombes ${ }^{48}$ (fig. 19). Une jonquière et une troisième île, en amont des précédentes, avaient été détruites par des dragages en 1842-1843, et les remblais rejetés le long de la rive gauche au nord du pont, à l'intérieur du clayonnage, long de plus de $1,5 \mathrm{~km}$ vers le sud, et qui prend appui dans sa partie nord sur les îles précitées. En 1869, pratiquement au niveau de nos découvertes de 1985, de nouveaux dragages le long de la rive gauche avaient déjà remonté un mobilier abondant. Il était peut-être issu des remblais de 1842-1843, mais sans doute aussi de zones non draguées de l'actuelle rive gauche. En effet, si les vases ou autre mobilier de faible taille purent être déplacés, ce ne pouvait pas être le cas des structures ; et, en 1869, " à une profondeur de cinq à six mètres au-dessous du niveau du sol, [la drague] se trouvait arrêtée [...] par des pièces de bois plantées ou gisant horizontalement ».J. Déchelette y a vu les restes de " constructions sur pilotis » qu'il est bien sûr tentant d'associer à des installations portuaires (Déchelette, 1913, p. 158).

47. Des carcasses de péniches visibles il y a deux ou trois décennies ont aujourd'hui irrémédiablement disparu dans la berge.

48. Cf. Bonnamour, 1976. Les différentes opinions des auteurs des $\mathrm{XIX}^{\circ}$ et $\mathrm{XX}^{c}$ s. y sont consignées, en particulier celle de L. Landa (1869), de J. Déchelette (1913) et L. Armand-Calliat (1937).

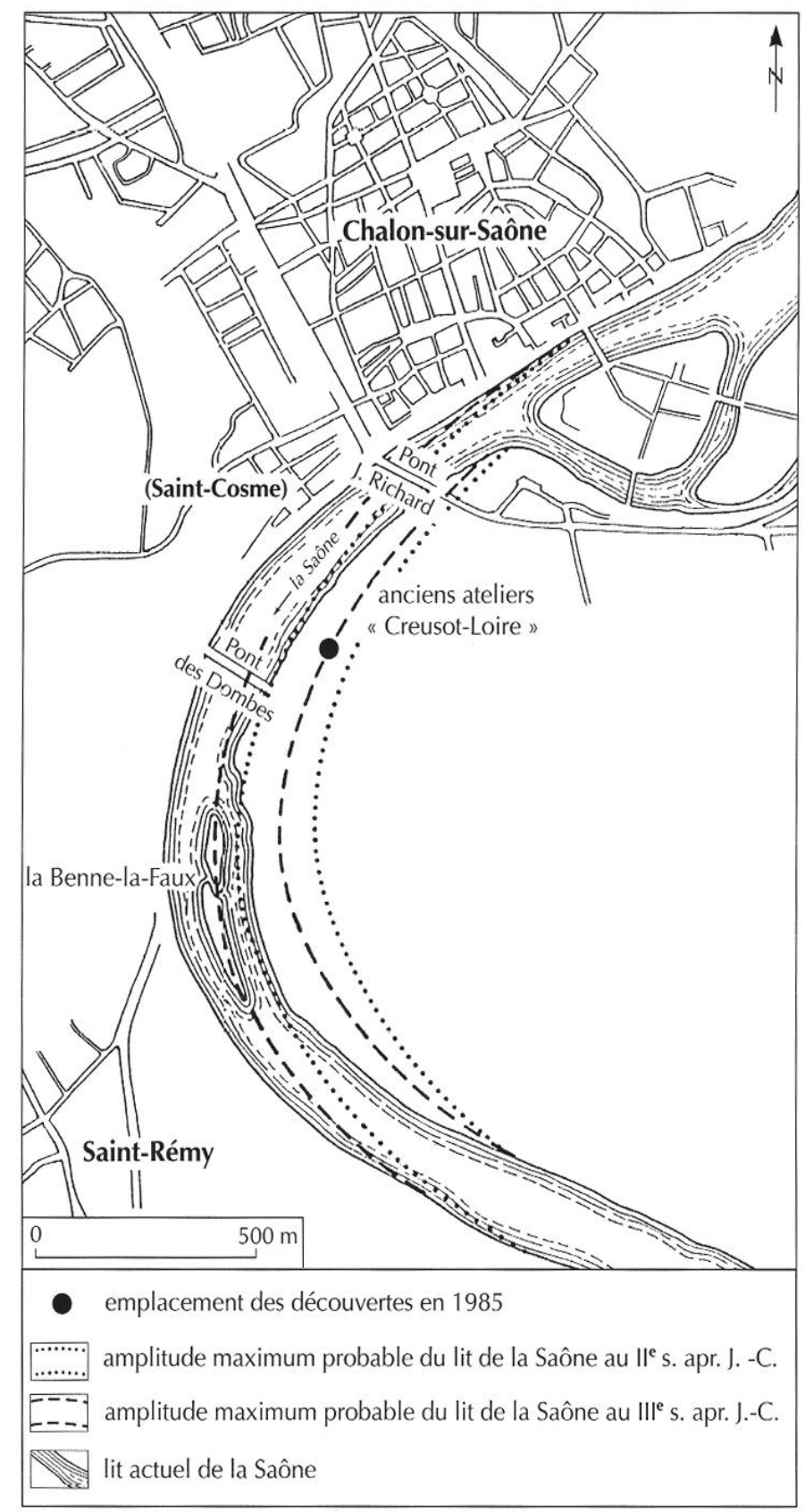

Fig. 19 - Plan de situation et d'interprétation des découvertes effectuées en 1985 au Petit-Creusot (dessin G. Monthel).

$\mathrm{Si}$ les découvertes du Petit-Creusot prouvent que la Saône s'est progressivement déplacée vers l'ouest, elles montrent aussi que la rive gauche pouvait être tout aussi occupée que la rive droite. Ces preuves d'activités commerciales et artisanales, jointes à la présence de la nécropole à environ $200 \mathrm{~m}$ du point fouillé, attestent que non seulement on travaillait ici 
mais qu'un habitat, même modeste, s'était certainement développé sur ce côté de la rivière. L'ensemble des îles, dont deux sont aujourd'hui conservées, et à l'ouest desquelles fut creusé le chenal actuel de la rivière, constituerait alors, dans cette hypothèse, les vestiges de l'ancienne rive droite de la Saône (fig. 19). Ces îlots débordés à l'ouest ont sans doute résisté à l'érosion par la présence d'éléments solidement implantés, que l'eau n'a pu arracher et qui, au contraire, ont piégé les sédiments ${ }^{49}$.

49. Sans doute le conseiller Millon était-il proche de la vérité, comme le rapporte J. Déchelette : "À cet endroit, la Saône décrit une courbe dont la rive gauche forme la partie concave. Par l'examen des lieux, le conseiller Millon a pu acquérir la conviction que les eaux, en accentuant cette courbe, ont fait de la rive droite d'autrefois la rive gauche d'aujourd'hui. " (Déchelette, 1913, p. 162). 


\section{BIBLIOGRAPHIE}

ANDRÉ J.

1985 : Les noms de plantes dans la Rome antique, Paris, Les Belles Lettres, $332 \mathrm{p}$.

\section{ANONYME}

1812a: [Mazade d'Avèze, J. B. D.], Itinéraire ou passe-temps de Lyon à Mâcon par la diligence d'eau, Lyon, J.-I. Maillet, VII + 171 p.

1812b: [Mazade d'Avèze, J. B. D.], Itinéraire ou passe-temps de Mâcon à Chalon par la diligence d'eau, Lyon, J.-L. Maillet, XI + 148 p.

\section{Armand-Calliat I..}

1937 : Le Chalonnais gallo-romain. Répertoire des découvertes archéologiques faites dans l'arrondissement de Chalon, Mémoires de la Société d'histoire et d'archéologie de Chalon-sur-Saône, XXVII, Chalon-sur-Saône, Société d'histoire et d'archéologie de Chalon-sur-Saône, 296 p.

\section{AUdOllent A.}

1923 : Les tombes gallo-romaines à inhumation des Martres-de-Vcyre, Mémoires de l'Académie des Inscriptions et Belles-Lellres, XIII, p. 275-328 [vannerie : p. 306-307].

\section{Aurigrmma A.}

1960 : L'Italia in Africa. Tripolitania II. I mosaici, Rome, Istituto poligrafico dello Stato, 77 p., 179 pl.

\section{BAIREM-BFN OSMAN W.}

1980 : Catalogue des mosaïques de Carthage, thèse de Doctorat de $3^{\text {e }}$ cycle, univ. d'Aix-en-Provence.

\section{BALFET $\mathrm{H}$.}

1952 : La vannerie. Essai de classification, L'Anthropologie, 56, 3-4, p. 259-280.

BANDI H. G.

1968 : Die Anfänge der archäologischen Sammlungen des Bernischen Historischen Museums in Bern, in:
Provincialia. Festschrift für Rudolf LaurBelart, Bâle, p. $511-522$.

\section{Baratte F., Painter K. (ÉdS)}

1989 : Trésors d'orfèrerie gallo-romains, Catalogue d'exposition, Paris, musée du Luxembourg, 8 fév.-23 avr. 1989, Paris, Réunion des musées nationaux.

Barbier G., Blanc N., Coulon G., GuRY F., PICHONNET M.

1999 : La vannerie à l'époque gallo-romaine, Catalogue d'exposition, SaintMarcel, musée d'Argentomagus, 26 juin-28 nov. 1999, musée d'Argentomagus, $63 \mathrm{p}$.

BACDRY F., BALI.FREAC L.

1873: Puits funéraires gallo-romains du Bernard (Vendée), La Roche-sur-Yon, Imprimeric L. Gasté, 359 p.

Bi.ANC N., Gury F.

1989 : Techniques de vannerie d'après les reliefs gallo-romains, in: Tïssage, corderie, vannerie, IX ${ }^{e}$ Rencontres intcrnationales d'Archéologie et d'Histoire, Antibes, oct. 1988, Juanles-Pins, éd. Al'DCA, p. 193-207.

1990a : Étude typologique des corbeilles de vendange sur les sculptures de Gaule romaine, in: Archéologie de la vigne et du vin, Caesarodunum, XXIV, p. 31-56.

$1990 \mathrm{~b}$ : La vannerie, artisanat traditionnel sur les mosaiques d'Afrique du Nord, in : L'Africa romana, Atti del VII convegno di studio, Sassari 1990, Sassari, ed. Gallizzi, p. 199-212.

Bi.ANChard-Lemée M., EnNaÏFer M., Slim H., SLim L.

1995 : Sols de l'Afrique romaine, Paris, Imprimerie nationale, $297 \mathrm{p}$.

BONNAMOUR I.

1976 : Le port gaulois et gallo-romain de Chalon, état de la recherche, Mémoires de la Société d'histoire el d'ar- chéologie de Chalon-sur-Saône, XLV (1975), p. 61-71.

BOUHLILA M.

1987 : Les mosaïques des thermes d'Ain Doura (Dougga, Tunisie), thèse de Doctorat de $3^{\mathrm{c}}$ cycle, univ. de ParisSorbonne IV.

Chevrier J.

1883: Chalon-sur-Saône pitloresque et démoli, environs el légendes, Paris, A. Quantin, $214 \mathrm{p}$.

Cleykt-Merle J.J.

1989 : Ia pêche préhistorique, in: Mohen J.-P. (ćd.), Le temps de la Préhistoire, vol. 2, Dijon, Société préhistorique française, éd. Archéologia, p. 69-71.

CROISIl.I.F. J.-M.

1965 : Les natures mortes campaniennes, coll. Iatomus, 76, Bruxelles, 135 p.

DÉCHLLLTTE J.

1913 : La collection Millon, antiquités préhistoriques et gallo-romaines, Paris, Paul Geuthner, 282 p., XIVI pl.

Desbat A.

1982 : Vases à médaillons d'applique des fouilles récentes de Lyon, Figlina, 5-6, Lyon, $207 \mathrm{p}$.

DUNBABIN K. M.

1978 : The Mosaics of Roman North Africa. Studies in Iconography and Patronage, Oxford Monographs on classical Archaeology, Oxford, Clarendon Press, 303 p.

ENNAÏFER M.

1973 : La civilisation tunisienne à travers la mosaïque, Tunis, Société tunisicnne de diffusion.

ESPÉRANDIEU É.

1907... : Recueil général des bas-reliefs de la Gaule romaine, Paris, Imprimerie nationale. 
FOUCHER L.

1960 : Inventaire des mosaïques, feuille $\mathrm{n}^{\circ} 57$ de l'Atlas archéologique, Sousse, Tunis, Institut national d'archéologie et arts.

FRAIIER G.

1994 : Mosaïques de Tunisie, Tunis.

\section{GAIT/SCH W.}

1986 : Anlike Korb-und Seilerwaren, Schriften des Limesmuseums Aalen, 38 , Aalen, 100 p.

Gasparri C.

1983 : Duc mosaici antichi in S. Maria in Trastevere, in: Alessandria $e$ il mondo ellenistico-romano. Studi in onore di Achille Adriani, III, Rome, «I'Erma » di Bretschneider, p. 672-676.

HaYes J. W.

1972 : Late Roman Pottery, Londres, British School at Rome.

1980 : Supplement to Lale Roman Pottery, Londres, British School at Rome.

HÖLSCHER F.

1992 : Ein Bild der Musse, in: Kotinos, Festschrift für F. Simon, Mayence, Philipp von Zabern, p. 211-213.

Ja)ul M., Goldsthin B.

1990 : La vannerie française, Paris, Réunion des musées nationaux, $315 \mathrm{p}$.

LANCHA J.

1981 : Recueil général des mosaïques de la Gaule -III- Province de Narbonncise - 2Vienne, $\mathrm{X}^{\mathrm{e}}$ suppl. à Gallia, Paris, éd. du CNRS, 320 p., $182 \mathrm{pl}$.

LANDA L.

1869 : Matériaux d'archéologie et d'histoire, Paris.

LALBENIHLIMER F.

1989 : Les amphores gauloises sous l'Empire: recherches nouvelles sur leur production et leur chronologie, in : Anfore romane e storia economica : un decennio di ricerche, Actes du colloque de Sienne, 22-24 mars 1986, École française de Rome, coll. de l'École française de Rome, 114, p. 105-138.
LEVI I).

1947 : Antioch mosaic pavements, Princeton, Princeton University Press, I.ondon, Oxford University Press, The Hague, H. Nijhoff, 2 vol., $650 \mathrm{p}$.

LINFERT A.

1974 : Eine Kanne für den Isis-Kult ? oder nur " nature morte" ?, Kölner Jahrbuch für vor- und frühgeschichte, 14, p. 17-27.

\section{Lissi-CaRONNA E.}

1995 : Un motivo decorativo, a rilievo applicato, della produzione della terra sigillata $\mathrm{C}$, Alli della Accudemia nazionale dei Lincei, Rendiconti classe di scienze morali, storiche e filologiche, ser. 9, 6/1, p. 185-194.

\section{MAICRI A.}

1958 : Ercolano. I nuovi scavi (1927-1958), Rome, Istituto poligrafico dello Stato, $509 \mathrm{p}$.

\section{MARGFRIN F.}

1994 : Le thème de la pêche dans la mosaïque romaine de Tunisie, mém. de Maîtrise d'histoire de l'art, univ. de Paris 1Panthéon-Sorbonne.

MrNatd M.

1992 : Artisans et centres de production de vannerie dans l'Occident romain, Mélanges de la Casa de Velasquez, 28, p. $58-63$.

\section{MONACCHI D.}

1996 : Un vilor e l'artigianato della cestineria ad Ameria, Mélanges de l'École française de Rome (Antiquité), 108, p. $943-977$.

MONTHEI. G.

1987 : Chronique archéologique, Chalon-sur-Saône, le Pctit-Creusot, Mémoires de la Société d'histoire el d'archéologie de Chalon-sur-Saône, 55 (19851986), p. $77-78$.

1987-1988 : Chalon-sur-Saône, Le Petit Creusot, in: Bourgogne, Gallia Informations, 1987-1988, 2, p. 39 et 41. 1996 : Chalon-sur-Saône, le PetitCreusot, lit fossile de la Saône aux II ${ }^{e}$ et $\mathrm{III}^{\mathrm{e}} \mathrm{s} .$, in: 30 ans d'archéologie en Saône-et-Loire, Catalogue d'exposition, Dijon, 1996, p. 296-298.

Monthel G., Scihoefer M., Valansot O., RFYER D. DF, NOWIK W.

1998 : I.es tissus gallo-romains de Chalon-sur-Saône, étude pluridisciplinaire, Centre international d'Étude des textiles anciens-Bulletin, 75, p. 21-36.

\section{MORDANT D.}

1987-1990: Noyen-sur-Seine avant le Néolithique: des vestiges mésolithiques en milieu humide, Bulletin $d u$ Groupement archéologique de Seine-etMarne, 28-31, p. 17-38.

1989 : Noyen-sur-Seine, Le Haut des Nachères (Seine-et-Marne), in: Archéologie de la France. 30 ans de découvertes, Catalogue d'exposition, Paris, Grand-Palais, 27 sept.-31 déc. 1989, Paris, Réunion des musées nationaux, notice 57 , p. 121.

POINSSOT C.

1965 : Quelques remarques sur la mosaïque de la maison de Dionysos et d'Ulysse à Thugga, in : La mosä̈que gréco-romaine, I, Colloque international du CNRS, Paris, 29 août3 sept. 1963, Paris, éd. du CNRS, p. $219-230$.

\section{ThOMASSON R.}

1992 : Sur la rive d'un ancicn méandre de la vieille Seine, une nasse de pêche en milieu celtique à Melz-surSeine (Seine-et-Marne), in: Actes du XIII colloque de l'Association francaise pour l'étude de l'âge du fer, Guéret, mai 1989, Limoges, p. 69-98.

VARONF. A.

1993 : Scavi recenti a Pompei lungo via dell'Abbondanza (Regio IX, ins. 12, 6-7), in : Ercolano 1738-1988. 250 anni di ricerca archeologica, Rome, "L'Erma » di Bretschncider, p. $617-640$.

Wuilleumirr P., Audin A.

1952 : Les médaillons d'applique de la iallée $d u$ Rhône, Paris, Les Belles Lettres, $189 \mathrm{p}$. 